\title{
HIC STUDET ATQUE LEGIT MONACHORUM CETUS ET ORAT. FORMA E FUNZIONE DEL CHIOSTRO NELLO SPAZIO DEL MONASTERO DALLE ORIGINI A CLUNY
}

\author{
ROBERTA CERONE
}

UDC: $726.71^{\prime \prime} 07 / 10^{\prime \prime}$

Original scientific paper

Manuscript received:30. 10. 2016.

Revised manuscript accepted: 27. 01. 2017.

DOI: 10.1484/J.HAM.5.113714
R. Cerone

Sapienza Università di Roma

Piazzale Aldo Moro 5

00195 Roma

Italia

The presence of the cloister in medieval monasteries is relatively late and, until the central Middle Ages, geographically limited to Central Europe. The analysis of material evidences attested until the 8th century the prevalence of settlement with multiple chapels scattered inside the enclosure alongside the more "orderly" type of monastery with the church and one or two residential wings arranged around a courtyard. From the 8th century begin to appear the first cloisters, but only in Central Europe, while the Mediterranean Europe delays even a few centuries. Starting from the 9th century the cloister spreads in the territories of the Empire, even in conjunction with the dissemination of the regula Benedicti, followed the reform of Benedict of Aniane. The cloister allowed a more rational layout of the buildings necessary for community life and ensured the total isolation of the monks. From the year 1000 and from the Cluny of Odilone's time, the monastery organized around the cloister lived a phase of strong experimentation, particularly with regard to the distribution of residential areas around the galleries. From the time of Cluny, the consecration of this type of setting can be reconstructed even through the different versions of the Consuetudines cluniacenses, that place in the cloister a multitude of activities and moments typical of the regular life.

Keywords: monastic architecture, cloister, St. Gall plan, Cluny, monastic customaries.

Nel chiostro di San Paolo fuori le mura a Roma una lunga iscrizione musiva su fondo oro ricorda, con la solenne evidenza della scrittura esposta, le funzioni principali del claustrum e ne esplica il significato simbolico in relazione all'osservanza della regola di san Benedetto, non rinunciando a sottolinearne l'eccezionale valore estetico' ${ }^{1}$ (fig. 1). Nonostante sia tarda in riferimento all'ambito cronologico del presente convegno, è particolarmente emblematica del ruolo che il chiostro - inteso come "lo spazio a corte che si trova chiuso tra l'edificio di culto e l'organismo residenziale, fornito di gallerie aperte a giorno che corrono lungo il perimetro dell'impianto" ${ }^{2}$ - ha assunto oramai in pieno Duecento nell'organizzazione dello spazio monastico e in rapporto alla vita regolare. Se il monumento ostiense segna dunque un punto d'arrivo, anche per l'eccezionale confezione formale e le scelte costruttive, individuare i presupposti dell'evoluzione claustrale è questione più controversa, complice la frammentarietà delle nostre conoscenze sugli esordi dell'architettura monastica e in particolar modo sull'evoluzione delle strutture residenziali annesse agli edifici di culto.
Definire la genesi e le modalità di diffusione del chiostro è un percorso indiziario che si avvale dell'ausilio delle fonti e dei dati, in costante aumento, forniti dall'archeologia. Senza entrare nel vivo della vexata quaestio relativa alle premesse che hanno generato il prototipo e che sono state di volta in volta individuate nell'atrio paleocristiano o nella villa romana con peristilio3, è oramai un dato assodato come la sua comparsa sia da collocare nell'ambito dell'VIII secolo e la sua propagazione da ricondurre al secolo ancora successivo e, almeno fino al 1000, da limitare all'Europa centrale ${ }^{4}$.

D'altronde già dal VI secolo risulta diffuso un tipo differente di impianto, il monastero/recinto di impostazione eremitica, che prevedeva all'interno dei septa monasterii una serie di fabbriche isolate, disposte con criteri diversi a seconda del contesto, ma comunque non a contatto le une con le altre. Emblematico è il modello sviluppatosi in Irlanda, mutuato secondo Legler dalle laure orientalis: un vallum circolare delineava lo spazio monastico e conteneva ulteriori recinzioni, a delimitare gli spazi accessibili ai vari livelli di utenza, nonché un numero variabile di oratori, di

“ "AGMINA SACRA REGIT LOCUS HIC QUEM SPLENDOR HONORAT / HIC STUDET ATQUE LEGIT MONACHORUM CETUS ET ORAT / CLAUSTRALES CLAUDENS CLAUSTRO DE CLAUDO VOCATUR / CUM CHRISTO GAUDENS FRATRUM PIA TURMA SERATUR / HOC OPUS EXTERIUS PRE CUNCTIS POLLET IN URBE / HIC NITET INTERIUS MONACHALIS REGULA TURBE / CLAUSTRI PER GIRUM DECUS AURO STAT DECORATUM / MATERIAM MIRUM PRECELLIT MATERIATUM / HOC OPUS ARTE SUA QUEM ROMA CARDO BEAVIT / NATUS DE CAPUA PETRUS OLIM PRIMITIAVIT / ARDEA QUEM GENUIT QUIBUS ABBAS VIXIT IN ANNIS / CETERA DISPOSUIT BENE PROVIDA DEXTRA IOHANNIS". Sul chiostro di San Paolo e la sua scansione cronologica v. E. BASSAN, L'architettura del monastero e il chiostro dei Vassalletto, in San Paolo fuori le mura a Roma, Firenze, 1988, p. 223-254; A.M. D'ACHILLE, Il chiostro di S. Paolo fuori le mura a Roma. Questioni aperte e prospettive di ricerca, in G. Villa (ed.), Storie di città e di architetture. Studi in onore di Enrico Guidoni, Roma, 2014, p. 395-414.

${ }^{2}$ P.F. PISTILLI, R. LEGLER, W. JACOBSEN, s.v., Chiostro, in Enciclopedia dell'arte medievale, IV, Roma, 1993, p. 694-718: 694.

3 Una completa bibliografia sul chiostro, seppur fino al 2004, è raccolta nell'appendice agli atti di convegno: P.K. KLEIN (ed.), Der mittelalterliche Kreuzgang - The medieval Cloister - Le cloître au Moyen Age. Architektur, Funktion un Programm, Regensburg, 2004, p. 392-408. Sull'origine dell'impianto si segnalano: J.-P. CAILLET, Atrium, péristyle et cloître: des réalités si diverse?, Ibidem, p. 57-65; R. LEGLER, Der abendländische Klosterkreuzgang - Erfindung oder Tradition?, Ibidem, p. 66-79. Ancora per una sintesi della questione v. P.F. PISTILLI, R. LEGLER, W. JACOBSEN, op. cit. (n. 2), in part. p. 694.

${ }^{4}$ In particolare, riguardo al periodo di esordio del modello claustrale, si segnalano i contributi di: W. JACOBSEN, Die Anfänge des abendländischen Kreuzgangs, in P.K. Klein (ed.), op. cit. (n. 3), p. 37-56; CH. SAPIN, De la cour au cloittre carolingien, in Les Cahiers de Saint-Michel de Cuxa, 46, 2015, p. 21-34; P.F. PISTILLI, Di chiostro in chiostro. Tipologia e sue declinazioni dalle origini alla forma monastica cistercense, in L. Ermini Pani (ed.), Gli spazi della vita comunitaria. Atti del Convegno internazionale di studio, Roma-Subiaco, 8-10 giugno 2015, Spoleto, 2016, p. 583-628: 589-604.

${ }^{5}$ R. LEGLER, op. cit. (n. 3), p. 75-76. 


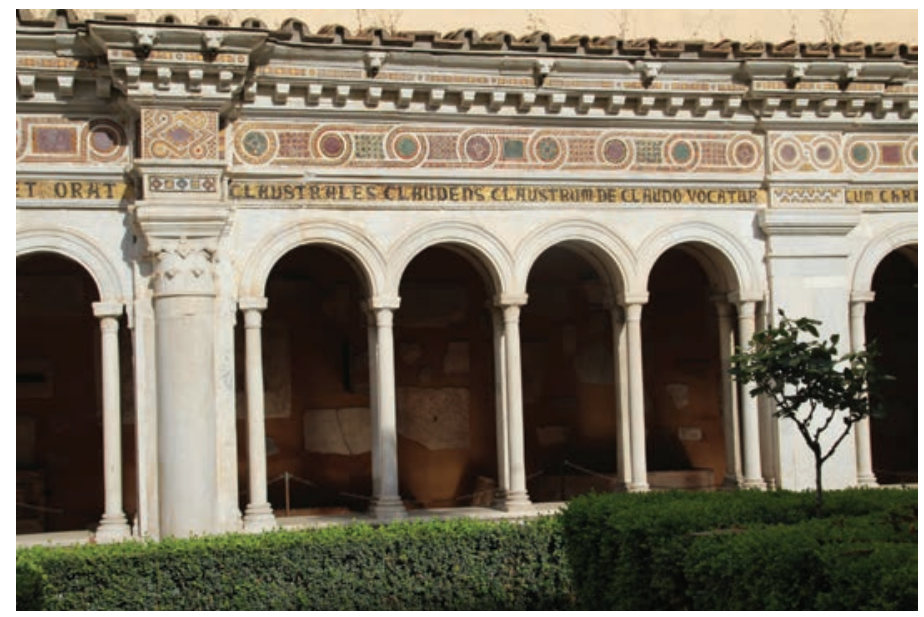

Fig. 1. Roma, San Paolo fuori le mura, chiostro, part. dell'iscrizione (foto Autore)

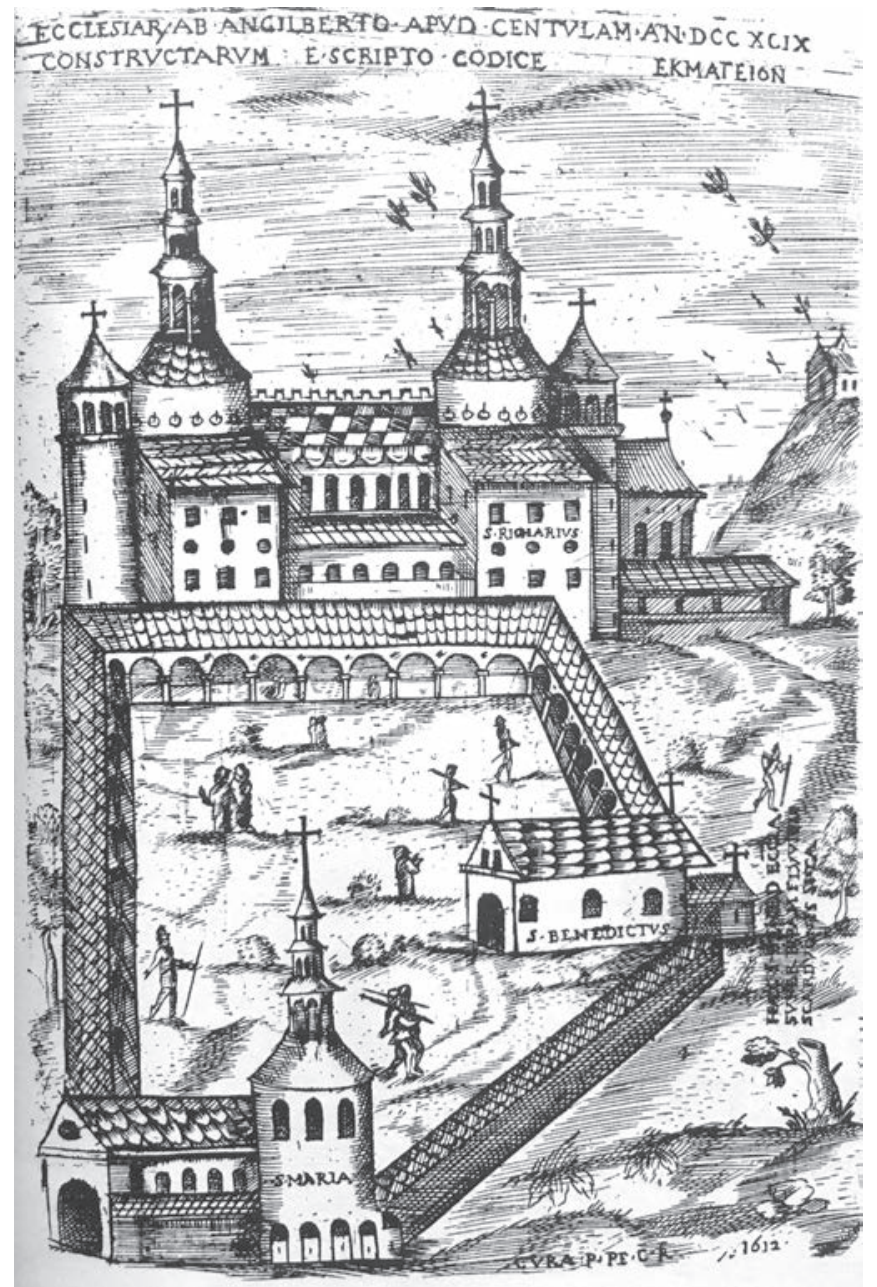

Fig. 3 Veduta del monastero di Centula/Saint Riquier (1612, da Heffman 1912)

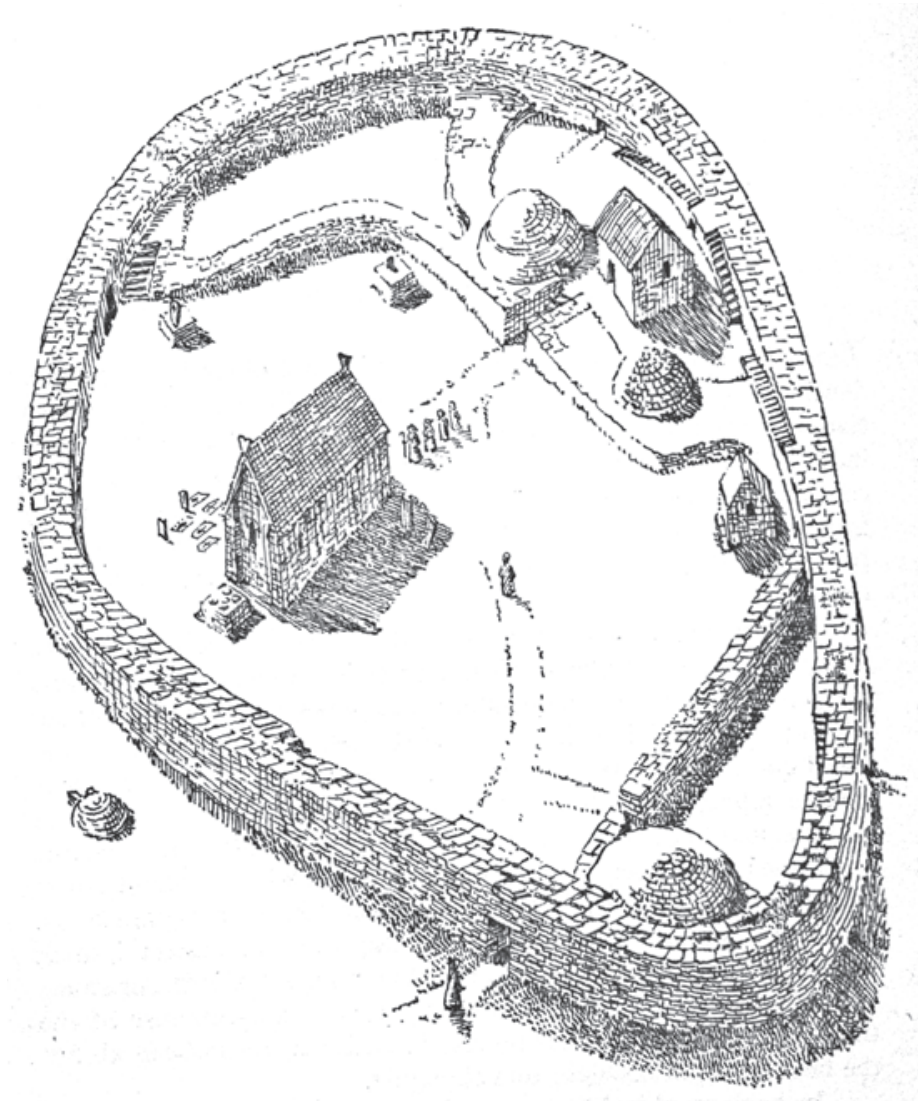

Fig. 2. Inishmurray, ipotesi restitutiva (da Horn 1973)

planimetria semplificata e dimensioni ridotte, mentre gli spazi comuni erano rappresentati da unità di pianta quadrata o circolare dove convivevano piccoli gruppi di confratelli ${ }^{6}$ (fig. 2). Variazioni sul tema si riscontrano sul continente dove alcuni monasteri franchi di particolare prestigio, come Jouarre, Nivelles, Manglieu ${ }^{7}$, registrano la compresenza di due o tre edifici di culto con diverse funzioni, in origine collegati tra loro da gallerie lignee (cosiddette longaniae) del tipo attestato poco più tardi a Centula/Saint Riquier e a Luxeuil $^{8}$ (fig. 3).

Meno conosciuta nel contesto merovingio è la conformazione delle strutture abitative, anche se i dati delle campagne di scavo più recenti cominciano $\mathrm{a}$ fornire indicazioni significative anche in questo senso, come il caso di Hamage nell'Hainaut che ben rappresenta la tendenza di molti monasteri continentali a evolversi verso un'organizzazione spaziale via via più compatta. Nel VII secolo Hamage presentava una scansione in celle distribuite sul versante sud della chiesa di Sainte-Marie in un'area delimitata da una palizzata e sostituite a partire dall'VIII secolo da ampi fabbricati in legno organizzati attorno a una corte centrale e dal

\footnotetext{
${ }^{6}$ W. HORN, Two early medieval monasteries, in P. Bloch (ed.), Intuition und Kunstwissenschaft. Festschrift für Hanns Swarzenski zum 7o. Geburtstag am 30. August 1973, Berlin, 1973, p. 53-66: 54-59; J.-M. PICARD, In platea monasterii: the layout of ecclesiastical settlements in early medieval Ireland (7 $7^{\text {th }}-9^{\text {th }}$ C.), in F. De Rubeis, F. Marazzi (eds.), Monasteri in Europa occidentale (secoli VIII-XI): topografia e strutture. Atti del Convegno Internazionale Museo Archeologico di Castel San Vincenzo 23-26 settembre 2004), Roma, 2008, p. 67-82; ID., L'organisation spatial des grandes monastères d'Irlande médiévale, in M. Lauwers (ed.), Monastères et espace social. Genèse et transformation d'un système de lieux dans l'Occident médiéval, Turnout, 2014, p. 213-225.

7 J. MERTENS, Recherches archéologiques dans l'abbaye mérovingiennes de Nivelles, in Miscellanea archaeologica in honorem J. Breuer, Bruxelles, 1962 , p. 89-113; E. JAMES, Archaeology and the Merovingian monastery, in H.B. Clarke, M. Brennan (eds.), Columbanus and Merovingian monasticism, Oxford, 1981 (BAR International Series, 113) p. 33-55; C. HEITZ, France Pré-romane. Archéologie et architecture religieuse du haut Moyen Âge; IVe siècle - an Mille, Paris, 1987 , p. 97-106.

${ }^{8}$ Sulla fisionomia e l'utilizzo delle gallerie monastiche di collegamento v. il recente S. BULLY, Circulation et hiérarchie au sein des établissements monastiques médiévaux: à propos de la grande galerie de l’abbaye de Saint-Claude (Jura), in M. Lauwers (ed.), op. cit. (n. 6), p. 353-375:365-372.
} 


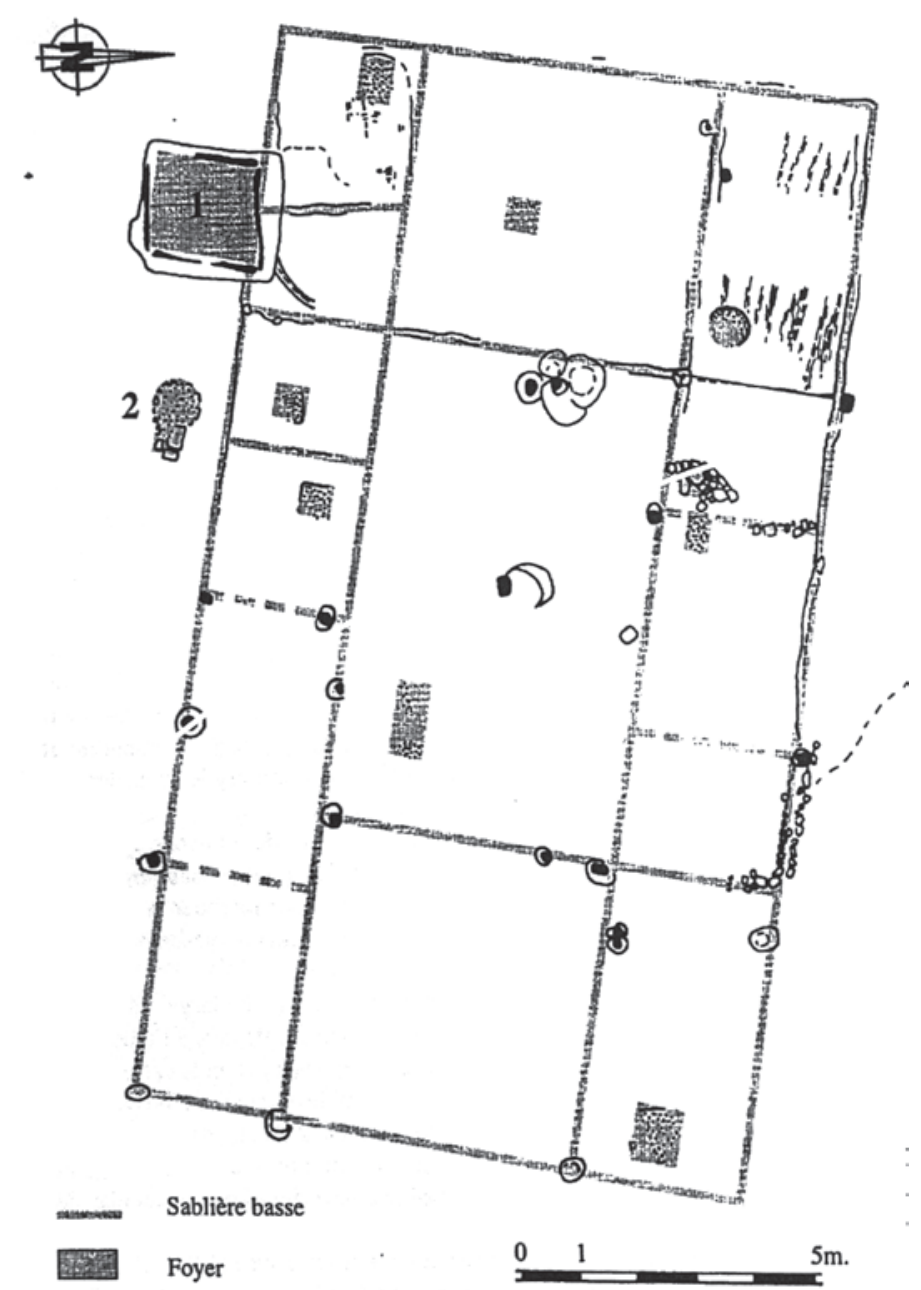

Fig. 4 Hamage, planimetria dell'edificio ligneo della fase IIIA (da Louis 1997)

carattere polifunzionale (fig. 4), non lontani dalle strutture attestate nella stessa epoca anche in altri siti come Sant'Afra ad Augsburg e Nôtre-Dame a Nevers'.

Nella sua evoluzione diacronica, Hamage registra dunque la transizione dalla forma monasterii a edifici sparsi all'impianto a unità raggruppate attorno a uno spazio aperto e il contestuale passaggio da una moltitudine di celle, a un edificio comunitario di ampie dimensioni. Questa tipolo-
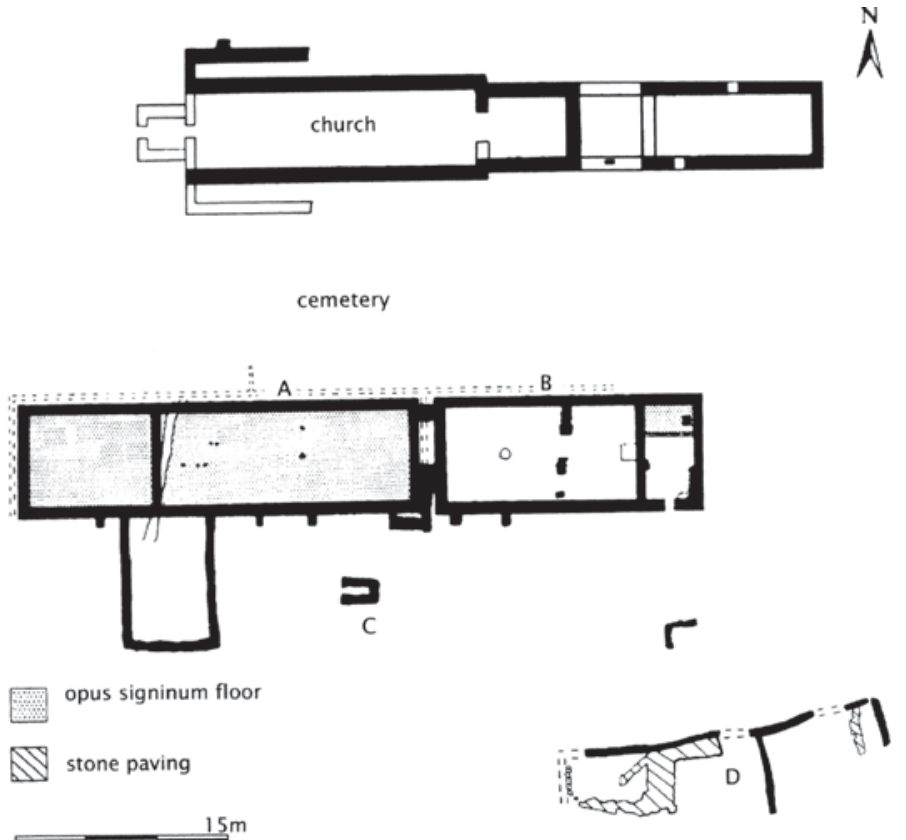

Fig. 5 Jarrow, planimetria ricostruttiva (da Cramp 1994)

gia trova anch'essa larga diffusione nell'Europa di VII-VIII secolo, con un raro antecedente nella penisola italiana, a San Sebastiano ad Alatri, dove la chiesa e una costruzione rettangolare si disponevano ai due lati opposti di uno spazio aperto $^{10}$. Nel contesto anglosassone tra VII e VIII secolo, i monasteri gemelli di Jarrow e Wearmouth in Northumbria declinano il tipo in maniera del tutto peculiare: attorno a una corte, in parte destinata a uso funerario, si fronteggiavano un oratorio doppio e un blocco ad esso parallelo per il quale, almeno in Jarrow, è ipotizzabile un uso residenziale ${ }^{\mathrm{n}}$ (fig. 5).

La mancanza di un modello dominante all'epoca delle regulae mixtae riflette la natura eterogenea del monachesimo occidentale prima dell'imposizione della regula Benedicti al tempo di Ludovico il Pio $(778-840)^{12}$. Fino ad allora nelle fonti non compaiono indicazioni costruttive, ma ci si limita a citare luoghi specifici - in particolare la chiesa, il refettorio, le cucine o il cellarium - per ambientare le attività dei confratelli e la parola claustrum, più spesso claustra, sta a indicare il cenobio nel suo complesso o, piuttosto, l'area della clausura e la separazione dal mondo esterno ${ }^{13}$.

\footnotetext{
${ }_{9}^{9}$ Gli edifici in legno che si susseguono nelle differenti fasi di Hamage, forse articolati su due livelli, presentavano dimensioni notevoli (più di 10 metri in pianta nei lati maggiori) e, al pianterreno, una ripartizione in ambienti centrali più ampi circondati da vani minori tra cui, almeno nella fabbrica riferita alla fase cd. IIIA uno spazio per le latrine, ma v., anche per gli altri monasteri citati, H. DANNHEIMER, Das coenobium beatae Afrae in Augsburg, in H.R. Sennhauser (ed.), Wohn- und Wirtschaftsbauten frümittelalterlicher Klöster. Internationales Symposium, 26.9.-1.10.1995 in Zurzach und Müstair, im Zusam-

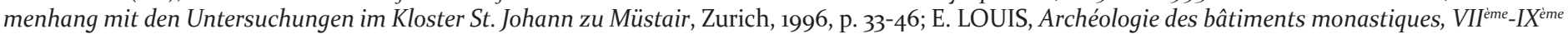
siècles; le cas de Hamage (France, Département du Nord), in G. de Boe, F. Verhaege (eds.), Religion and belief in Medieval Europe. Papers of the 'Medievale Europe Brugge 1997' Conference, Zellik, 1997, p. 55-63; ID., Hamage (nord). Espaces et bâtiments claustraux d'un monastère mérovingien et carolingien, in R.-H. Bautier (ed.), Pratique et sacré dans les espaces monastiques au Moyen Age et à l'époque moderne. Actes du colloque de Liessies-Maubeuge, 26, 27 et 28 septembre 1997, Amiens, 1998, p. 73-97; ID., Espaces monastiques sacrés et profanes à Hamage (Nord), VIIe-IXe siècles, in M. Lauwers (ed.), op. cit. (n. 6), p. 435-471; CH. SAPIN, L'archéologie des premiers monastères en France (Ve-déb. XI ${ }^{e}$ s.), un état des recherches, in F. De Rubeis, F. Marazzi (eds.), op. cit. (n. 6), p. 83-102.

${ }^{10}$ Questa fase è ricondotta al VI secolo in: E. FENTRESS, The sixth century abbey, in E. Fentress, (ed.), Walls and memory. The Abbey of San Sebastiano at Alatri (Lazio) from late Roman monastery to Renaissance villa and beyond, Turnhout, 2005, p. 33-70.

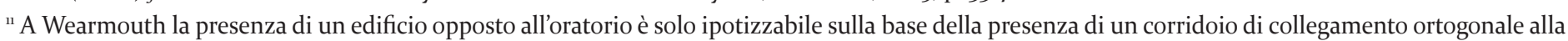
chiesa, mentre sono noti maggiori dettagli della fabbrica rettangolare in Jarrow che forse alloggiava il refettorio e un locale destinato al lavoro, v. R. CRAMP, Monkwearmouth and Jarrow in their Continental Context, in K. Painter (ed.), Churches built in ancient times. Recent studies in early Christian archaeology, London, 1994, p. 279-293; EAD., Monastic settlements in Britain in the $7^{\text {th }}-11^{\text {th }}$ centuries, in F. De Rubeis, F. Marazzi (eds.), op. cit. (n. 6), p. 113-133.

${ }^{12}$ G. ANDENNA, C. BONETTI (eds.), Benedetto di Aniane: vita e riforma monastica, Roma, 1993; J. SEMMLER, Karl der Große und das fränkische Mönchtum, in B. von Bischoff (ed.), Karl der Große, Lebenswerk und Nachleben, II, Das geistige Leben, Düsseldorf, 1965, p. 255-189.

${ }^{13}$ Fondamentale per l'indagine sulle fonti è P. BONNERUE, Eléments de topographie historique dans les règles monastiques occidentales, in Studia monastica, 37, 1995, p. 57-77, ma si vedano anche i più recenti: S. UGGÉ, Lieux, espaces et topographie des monastères de l'Antiquité tardive et du haut Moyen Âge.
} 


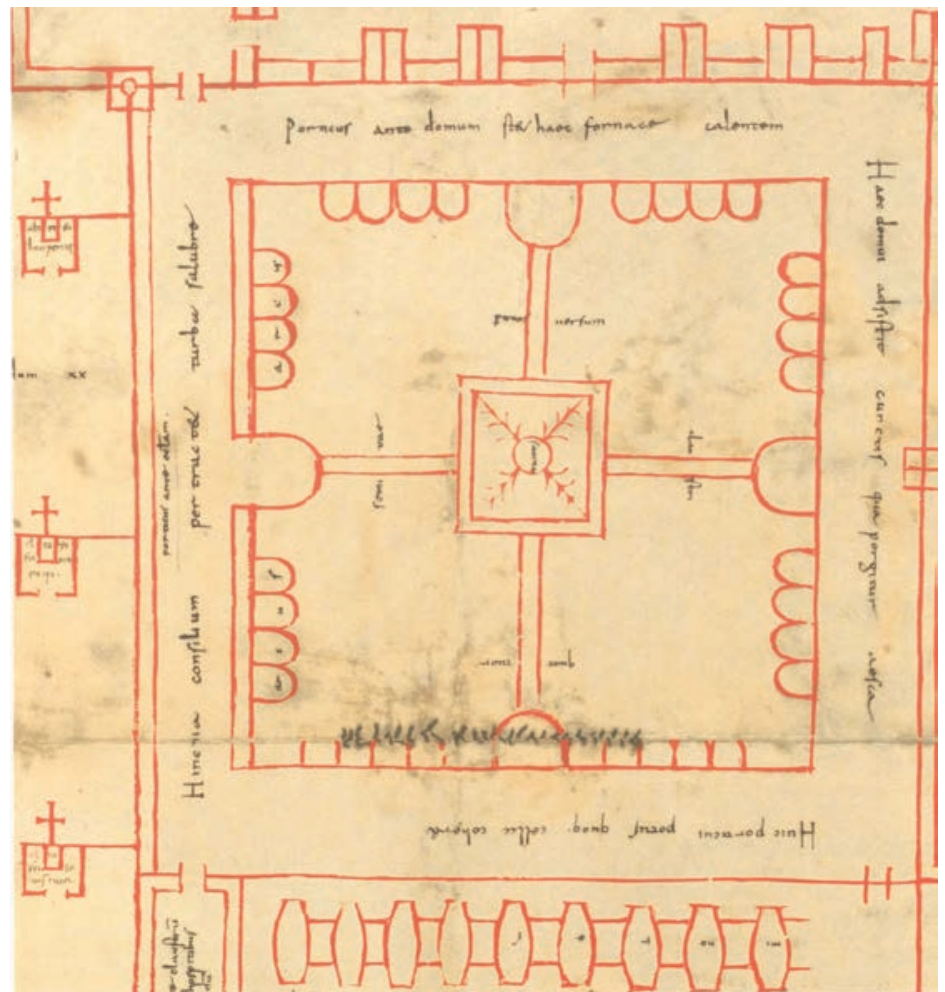

Fig. 6 St. Gallen, Stiftsbibliothek, Piano di San Gallo, chiostro (da Horn, Born 1979)

Per trovare un sicuro riferimento al quadrato porticato occorre attendere il IX secolo quando, però, l'ambiguità del termine non è stata ancora completamente superata nella letteratura monastica, tanto da spingere verso la metà del secolo Ildemaro di Corbie a chiarire al lettore il significato di claustra monasterii, vale a dire "quella corte riservata ai monaci che sta tra un porticato e l'altro" ${ }^{14}$. Nell'Expositio Ildemaro allude dunque per la prima volta in maniera inequivocabile al chiostro, descritto nei suoi elementi caratterizzanti - lo spazio aperto e le gallerie - e ne propone l'identificazione con l'officina dove, secondo i dettami di Benedetto, il monaco deve esercitare gli instrumenta delle buone opere, non rinunciando a consigliare l'ampiezza ottimale (10o piedi) perché il porticato si adatti alla vita regolare senza offrire occasioni di distrazione ${ }^{15}$.

Le osservazioni dell'autore, che si dilunga anche sugli altri spazi della vita comune commentando differenti consuetudini ${ }^{16}$, si collegano all'eccezionale esperienza di vita del personaggio che, nei suoi viaggi per i territori dell'Impero e

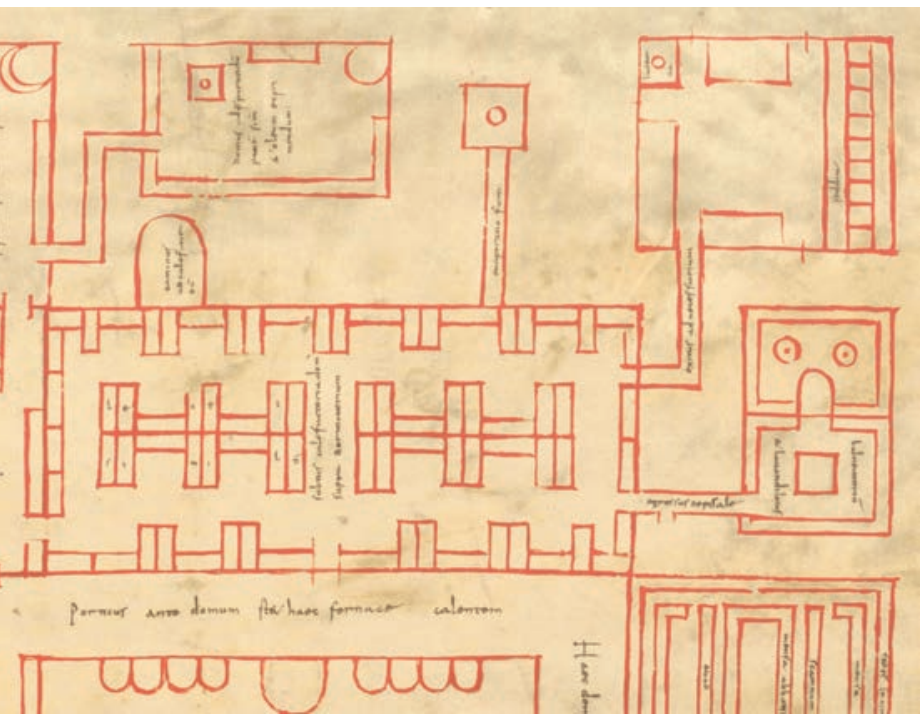

Fig. 7 St. Gallen, Stiftsbibliothek, Piano di San Gallo, dormitorio (da Horn, Born 1979)

prima del suo insediamento definitivo a Civate, ebbe l'occasione di visitare numerosi centri monastici ${ }^{17}$, tra cui alcuni cenobi che già all'epoca erano articolati secondo lo schema ad ali porticate, come San Faustino Maggiore di Brescia e San Salvatore nella medesima città, Reichenau e San Gallo. Proprio questi ultimi costituiscono uno snodo fondamentale nella parabola claustrale, poiché è in tale ambito che si conservano le prime tracce materiali di un monastero organizzato attorno a un claustrum, come si vedrà più avanti, ma soprattutto è qui che, pochi decenni prima del commento di Ildemaro, il modello viene cristallizzato nel Piano di San Gallo, concepito su probabile iniziativa dell'abate di Reichenau Haito (8o6-823) come dono per l'abate di San Gallo Gozberto $(816-837)^{18}$.

Il celebre disegno mostra la planimetria di un monastero ideale in cui il chiostro è lo strumento organizzativo del cuore della clausura e, in una formulazione doppia di minori dimensioni, anche delle aree più esterne, dedicate agli infermi e ai novizi. La corte è inserita subito a destra dell'abbaziale ed è definita da ambulacri aperti da coppie di quadrifore inquadranti aperture centrali, poste in corrispondenza dei sentieri che attraversano la corte (fig. 6). Lo schema a blocchi ortogonali denominati domus nei tituli, che serrano il cortile, mostra il definitivo superamento del sistema a celle e fabbricati isolati, pur recuperando un tipo di edificio bilivello di notevoli dimensioni e dal carattere

Réflexions à propos des règles monastiques, in M. Lauwers (ed.), op. cit. (n. 6), pp. 15-42; F. MARAZZI, La città dei monaci. Storia degli spazi che avvicinano a Dio, Milano, 2015, p. 88-113.

${ }^{14}$ R. MITTERMÜLLER (ed.), Expositio Regulae ab Hildemaro tradita, in Vita et regula ss. P. Benedicti una cum expositione Regulae a Hildemaro tradita, III, Ratisbonae, Neo-Eboraci et Cincinnati, 1880.

${ }_{15} \mathrm{Ibidem}$, p. 182-184: "Tutte le attività del monaco devono svolgersi nel chiostro dove possono essere compiute senza peccato (...) e questo deve essere costruito grande abbastanza da contenere tutto ciò che serve al monaco per le sue attività, dove possa lavare i panni, leggere nel tempo libero (...) e cose simili. Perché se il chiostro è più grande del dovuto, il monaco può incontrarci un laico o un estraneo, con cui può parlare o a cui può dare e da cui può ricevere qualcosa senza il permesso dell'abate trovando così occasione di peccare. Allo stesso modo, nel caso non sia sufficiente, cioè piccolo per il bisogno, allora ci sarà la tentazione di uscire (...) L'abate deve organizzare il chiostro in maniera tale che si possa esercitare la stabilitas e che non ci sia alcuna occasione di perdere tempo. Molti sostengono che il chiostro monastico debba essere ampio cento piedi in ogni parte, non di meno altrimenti sarebbe piccolo". Sul testo e sull'autore v. G. ARCHETTI, Spazi e strutture claustrali nei commenti carolingi alla regola benedettina, in Hortus artium medievalium, 20, 2014, 2, p. 448-462; ID., "Dilexi decorem domus tuae". Committenza aristocratica e popolare in ambito claustrale (secoli VIII-XII), in A.C. Quintavalle (ed.), Il Medioevo: i committenti, Atti del Convegno internazionale di studi (Parma, 21-26 settembre 2010), Milano 2011 (I convegni di Parma, 13), pp. 237-251.

${ }^{16}$ Ibidem, p. 450.

${ }^{17}$ Ibidem.

${ }^{18}$ Sul Piano di San Gallo sono tuttora fondamentali gli studi di: W. HORN, E. BORN, The plan of St. Gall: a study of the architecture and economy of, and life in a paradigmatic carolingian monastery, 3 voll., Berkeley, 1979 (California Studies in the History of Art, 19); W. JACOBSEN, Der Klosterplan von St. 


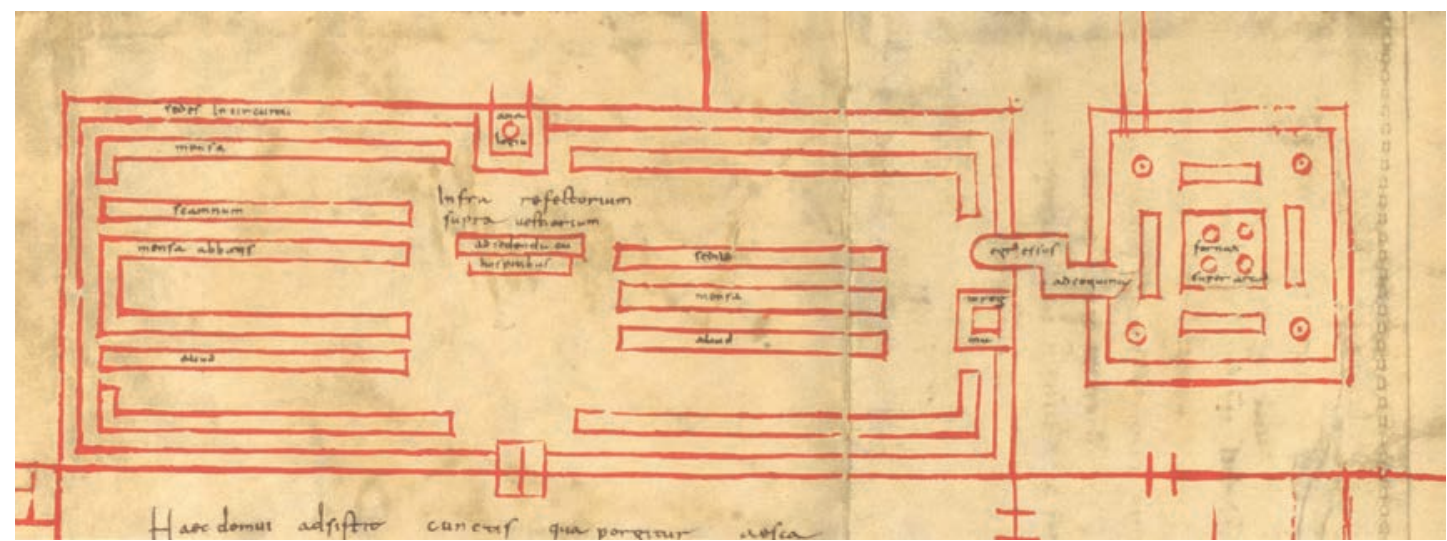

Fig. 8 St. Gallen, Stiftsbibliothek, Piano di San Gallo, refettorio (da Horn, Born 1979)

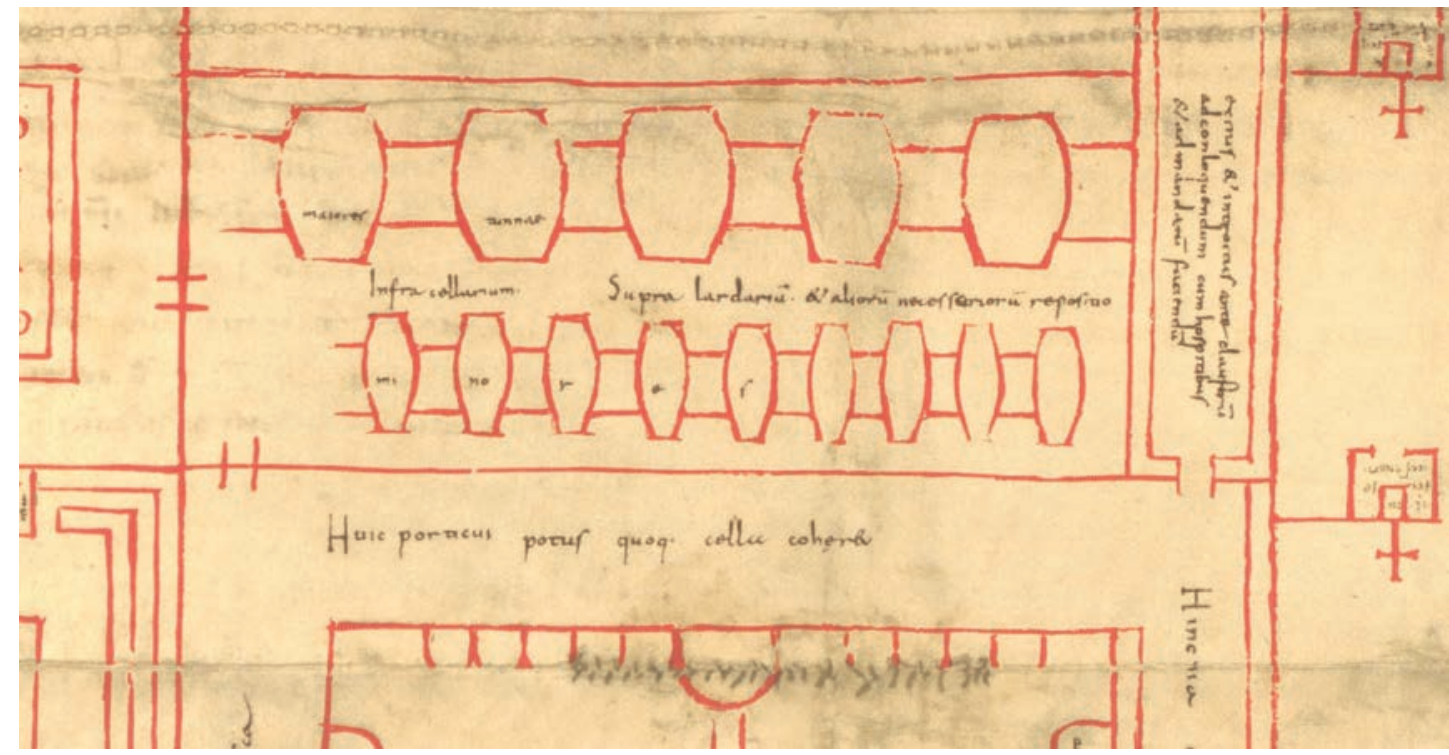

Fig. 9 St. Gallen, Stiftsbibliothek, Piano di San Gallo, cellarium (da Horn, Born 1979)

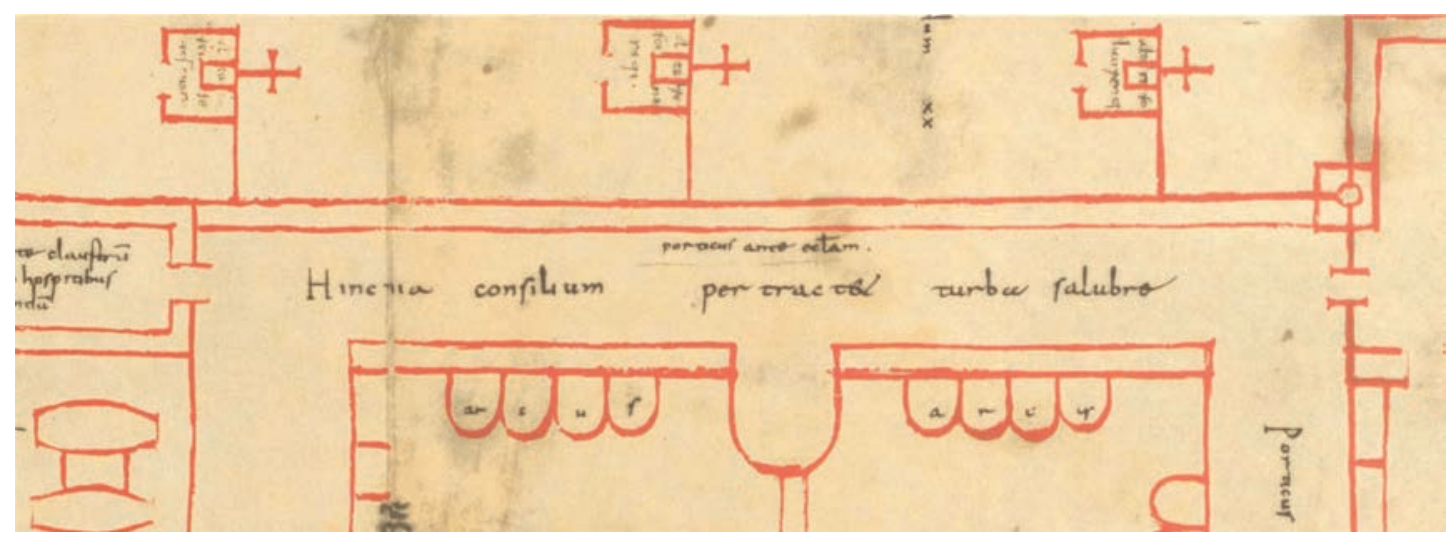

Fig. 10 St. Gallen, Stiftsbibliothek, Piano di San Gallo, galleria del chiostro utilizzata per il Capitolo (da Horn, Born 1979)

multifunzionale i cui immediati precedenti sono individuabili negli ampi caseggiati che, come già visto per Hamage, l'archeologia sta riportando alla luce in numerosi siti. Nel settore superiore, a contatto con il presbiterio, il blocco a due piani contiene il calefactorium al piano terra e, al di sopra, il dormitorio (fig. 7). Nell'ala opposta alla chiesa trovano posto il refettorio collegato alla cucina e sormontato dal vestiarium e infine, nella fabbrica inferiore, il cellarium con al livello superiore la dispensa dei vini (figg. 8-9). Gli ambulacri permettono un collegamento rapido e al riparo dalle intemperie tra i vari ambienti e sono ad essi collegati dal punto di vista funzionale, come esplicano le stesse didascalie. Particolare rilievo assume la galleria adiacente la chiesa, dotata di bancalia descritti da una doppia linea rossa, utili ad accogliere l'officio capitolare, come suggerisce il titulus (Hinc pia consilium pertractet turba salubre), in un

Gallen und die Karolingische Architektur, Berlin, 1992. Si vedano altresì i più recenti: H.R. SENNHAUSER, À propos de l'architecture monastique, entre Saint-Gall et Cluny II, in D. Iogna-Prat, M. Lauwers, F. Mazel, I. Rosé (eds.), Cluny. les moines et la société au premier âge féodal. Actes du colloque en 2 volets, Romainmôtier, 24- 26 juin 2010 et Cluny, 9 - 11 septembre 2010, Rennes, 2013, p. 527-547; P. ERHART, La pianta di San Gallo: un archivio di spazi monastici, in L. Ermini Pani (ed.), op. cit. (n. 4), p. 1-35. 


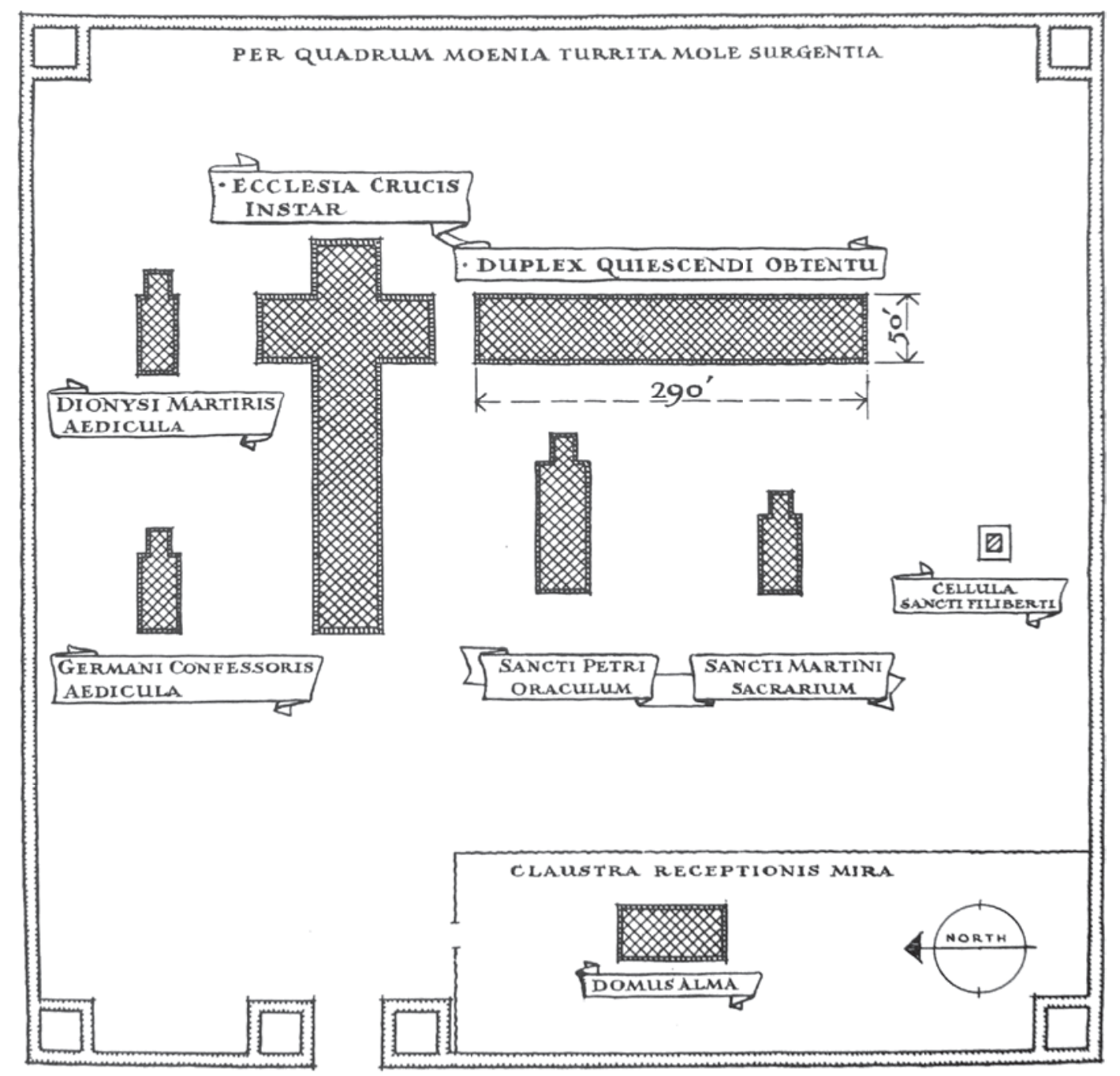

Fig. 11 Gemeticum/Jumièges, ipotesi restitutiva (da Horn 1973)

momento in cui nell'assetto del monastero ancora non si registra la presenza costante di una sala specifica ${ }^{19}$ (fig. 10).

Il Piano di San Gallo elegge il quadrato claustrale a insediamento esemplare in un momento in cui, del resto, anche l'archeologia ne attesta l'avvenuta diffusione. Se da un lato la propagazione del modello coincide a livello temporale con il rinnovamento del mondo monastico innescato dai sinodi di Aquisgrana, l'assenza di una teorizzazione esplicita lascia pensare che fu piuttosto la prassi costruttiva a determinare l'accelerazione del fenomeno. Il chiostro, d'altronde, permetteva una disposizione razionale degli edifici necessari alla vita comune, garantendo al contempo il totale isolamento della comunità e doveva pertanto sembrare garante per una vita in piena aderenza alla Regola, in particolare nei centri monastici più grandi. Gli effetti della riforma anianea si sentirono soprattutto nelle regioni centrali dell'Impero ed è proprio qui, nei cenobi di maggior prestigio, che lo schema aveva attecchito già da un secolo, anche se non è possibile definirne l'esatta genealogia, né delineare le direttrici di sviluppo.
A questo proposito, l'ipotetica primogenitura di Gemeticum/Jumièges rappresenta una questione di non facile risoluzione $^{20}$. La Vita Philiberti, redatta dal monaco Ermentaire all'inizio del IX secolo sulla base di un testo precedente, descrive diffusamente il cenobio, ma non è dato stabilire con certezza se l'autore si riferisca davvero all'abbazia del tempo di san Filiberto (la metà del VII secolo), come egli afferma, o se invece sia la compagine a lui contemporanea l'oggetto della sua rassegna ${ }^{21}$. In ogni caso, poi, la significativa attestazione di un blocco residenziale a due piani (duplex quiescendi obtentu), che come nel Piano di San Gallo conteneva refettorio e al di sopra il dormitorio, si accompagna a un più oscuro riferimento alla corte porticata cui la frase "operosa saxis claustra comitantur arcus" sembra alludere e su cui però non esiste al momento alcuna evidenza (fig. 11). Né è indicativa la presenza precoce di un chiostro attorno al 710 nel monastero di Palatiolum-Pfalzel presso Treviri che si impianta in una villa rustica di epoca romana e che dunque reimpiega all'uopo il peristilio interno ${ }^{22}$.

\footnotetext{
${ }^{19}$ Sulla problematica collocazione della sala capitolare negli esordi dell'architettura monastica mi permetto di rimandare a: R. CERONE, «Regula in capitulo pronunciata fuerit». La sala capitolare nei monasteri medievali (secc. IX-XIII), in in L. Ermini Pani (ed.), op. cit. (n. 4), p. 51-83.

${ }^{20} \mathrm{~N}$. MANOURY, Les bâtiments conventuels de l'abbaye de Jumièges (VII ${ }^{-}$-XVIII ${ }^{e}$ s.), in Archéologie médiévale, 26, 1996, p. 77-107; J. LE MAHO, Le monastère de Jumièges (France) aux temps mérovingiens (VII ${ }^{e}$-VIII ${ }^{e}$ siècle): les témoignages des textes et de l'archéologie, in HAR, 9, 2003, p. $315-322$.

${ }^{21}$ L. D'ACHERY, J. MABILLON (eds.), Vita sancti Filiberti, in Acta Sanctorum Ordinis sancti Benedicti, II, Paris, 1669, p. 819. Sulle diverse ipotesi cronologiche relative alla descrizione di Jumièges, v. J. LE MAHO, op. cit. (n. 20), p. 315-316.

${ }^{22}$ H. CÜPPERS, Palatiolum-Pfalzel, in T. Kempf, W. Reusch (eds.), Früchristliche Zeugnisse im Einzugsgebiet von Rhein und Mosel, Trier, 1965, pp. 152-162.
} 

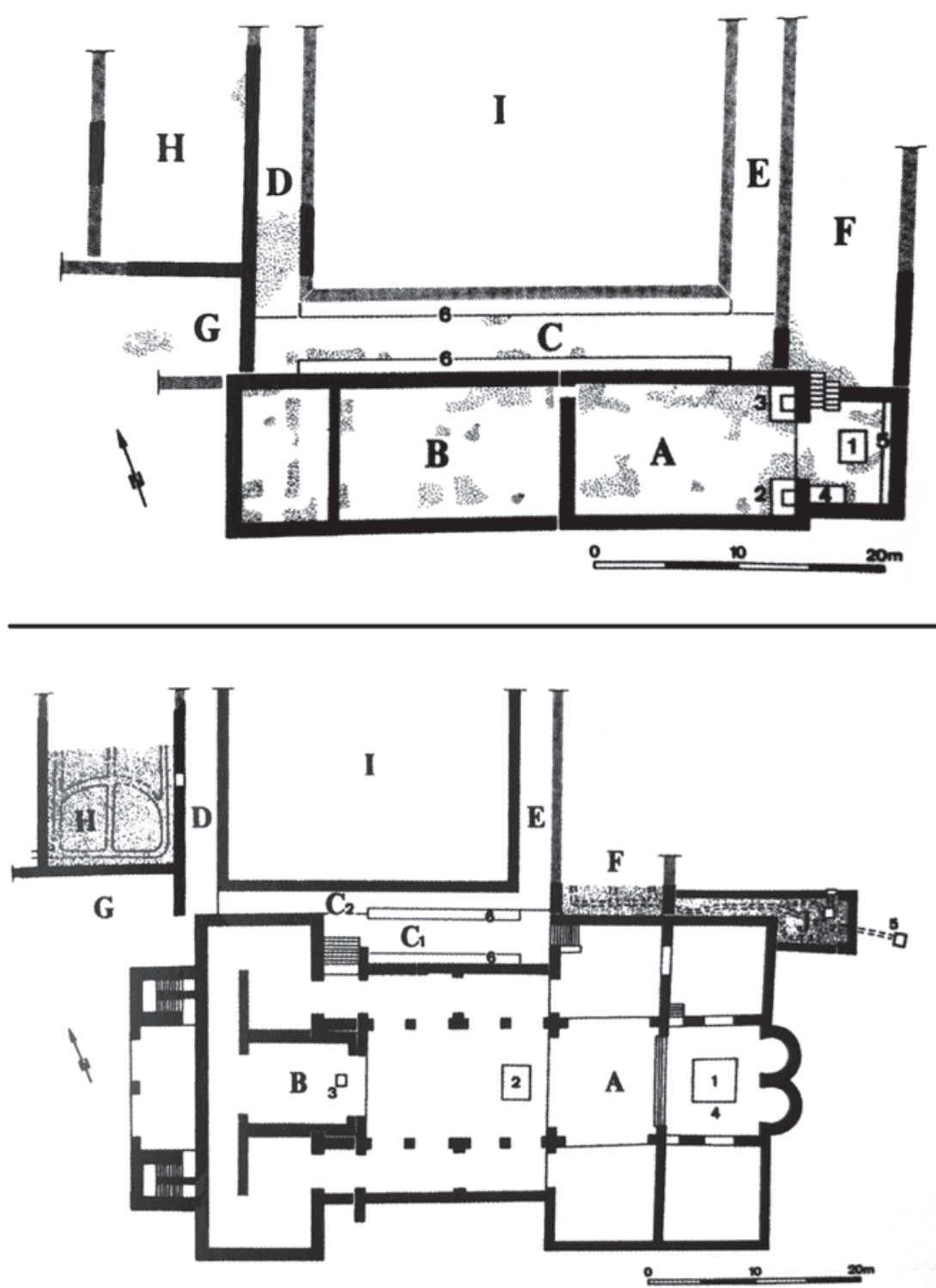

Fig. 12 Reichenau-Mittelzell, planimetrie restitutiva del complesso tra VIIIe X secolo (da Zettler) ca.), incubarono lo schema con la chiesa abbaziale e gli edifici residenziali a delimitare il quadriportico che, almeno in Lorsch I, sul lato opposto all'abbaziale era semplicemente chiuso da un muro di cinta. L'esatta destinazione delle imponenti domus attorno allo spazio centrale non è sempre definibile, ma se in Lorsch I le ali est e ovest sono ripartite in tre vani dalle funzioni imprecisabili ${ }^{25}$, almeno nel caso della Mittelzell sembra che la fabbrica tangente al coro (F) ospitasse una sala dei monaci nel vano unico al pianterreno e, al di sopra, il dormitorio collegato con il coro tramite una rampa di scale, mentre dirimpetto si ipotizza la presenza del cellarium in una situazione analoga al piano di San Gallo, come del resto accade anche per l'utilizzo per l'officio capitolare della galleria adiacente la chiesa, per questo dotata di bancali allineati $(C)^{26}$.

Ancora entro l'VIII secolo accolsero l'organismo claustrale nuove fondazioni come St. Alban a Magonza o Schwarzach ${ }^{27}$, mentre altrove furono compagini già esistenti, spesso installate su preesistenze romane, a essere rimodulate all'insegna del chiostro, come Schuttern o Herrenchiemsee $^{28}$ (fig. 14). Di quest'ultima, seguendo Dannheimer, è possibile ripercorrere le diverse fasi di aggregazione, dalla rifondazione nel $745 \mathrm{ca}$. alla sua distruzione nel 907 a opera degli Ungari, e seguire, come nella Mittelzell, il passaggio dall'impiego di caseggiati lignei distribuiti ad angolo retto - senza che gli edifici fossero in contatto l'uno con l'altro e senza che ci fossero già in questa fase delle gallerie a definire il cortile - a fabbriche in muratura prospicienti porticati, la cui distribuzione fuori asse attesta la faticosa genesi del sito ${ }^{29}$.

A partire dal IX secolo la propagazione

Per trovare spazi a quattro ali progettati ex novo occorre spingersi fino a quasi la metà dell'VIII secolo e spostarsi a ridosso dell'arco alpino con la Reichenau-Mittelzell sul lago di Costanza ${ }^{23}$, seguita dopo qualche decennio dalla cosiddetta Altenmüster di Lorsch, o Lorsch I, in Assia ${ }^{24}$ (figg. 12-13). Entrambe le abbazie, rispettivamente durante i ministeri di Pirminus (734-727 ca.) e Gundeland (766 dell'impianto claustrale nell'impero carolingio finì per interessare pressoché tutte le maggiori abbazie, ma dal rinnovamento in atto, che si protrasse in età ottoniana, rimasero fuori intere aree geografiche e in particolare, almeno allo stato attuale delle conoscenze, gran parte della penisola italiana, compresa la città di Roma che fino al XII secolo non presentava alcun monastero con siffatto impianto ${ }^{30}$.

\footnotetext{
${ }_{23}$ Il primo chiostro della Mittelzell era in legno, ma fu ricostruito in muratura forse già al tempo dell'abate Arnefrido (734-746), ma v. A. ZETTLER, Die frühen Klosterbauten der Reichenau. Ausgrabungen, Schriftquellen, St. Gallen Klosterplan, Sigmaringen, 1988, (Archäologie und Geschichte. Freiburger Forschungen zum ersten Jahrtausend in Südwestdeutschland, 3), p. 166-174; ID., Die Konventbauten der Klösterlichen Niederlassungen auf der Reichenau, in H.R. Sennhauser (ed.), op. cit. (n. 9), p. 271-276.

${ }^{24}$ F. BEHN, Die karolingische Klosterkirche von Lorsch an der Bergstrasse nach den Ausgrabungen von 1927-1928 und 1932-1933, I, Berlin-Leipzig, 1934, p. 17-20.

${ }^{25}$ Ibidem, p. 17.

${ }^{26}$ A. ZETTLER, Die Konventbau, op. cit. (n. 23), p. 275.

${ }_{27}^{27}$ E. NEEB, Zur Baugeschichte der St. Albanskirche bei Mainz, in Mainzer Zeitschrift 3, 1908, p. 69-100; W. JACOBSEN, op. cit. (n. 4), p. 47-49.

${ }^{28}$ K. LIST, Die Reichabtei Schuttern. Ergebnisse der Grabungen in den Jahren 1972 bis 1975, in Denkmalpflege in Baden-Württemberg, 4, 1975, 3, p. 107-116; H. DANNHEIMER, Die Klöster auf dem Chiemsee-Inseln, in Pfalz - Kloster - kloster-pfalz. St. Johann in Müstair, in H.R. Sennahauser (ed.), Historische und archäologische Fragen, Zürich, 2010, p. 127-138.

${ }^{29}$ Ibidem.

${ }^{30}$ Sul "ritardo" della penisola italiana si rimanda a: P.F. PISTILLI, Il chiostro e l'abbazia: insediamenti monastici nell'Italia altomedievale, in A.C. Quintavalle (ed.), Medioevo mediterraneo: l'Occidente, Bisanzio e l'Islam. Atti del convegno internazionale di studi, Parma, 21 - 25 settembre 2004, Milano, 2007 (I convegni di Parma, 7), p. 294-303. Mentre sul caso di Roma: R. CERONE, Il chiostro a Roma tra XII e XIII secolo: genesi, evoluzione e diffusione di un modello, in J. Ramôa Melo, G. Rossi Vairo (eds.), Encontro internacional sobre claustros no mundo mediterrânico (séculos X-XVIII), Coimbra, 2016, p. 41-54.
} 


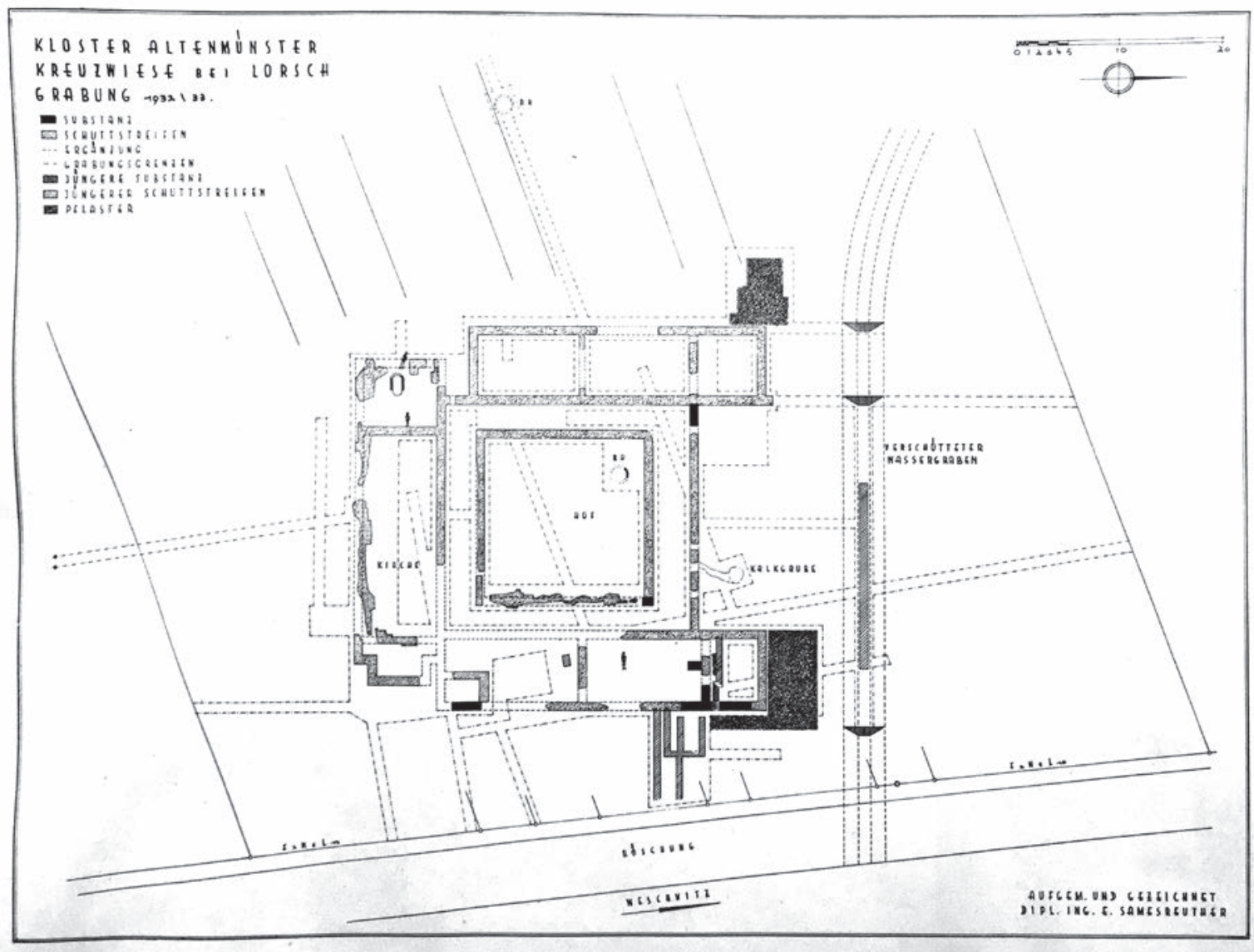

Fig. 13 Lorsch, Altenmünster (Lorsch I), planimetria ricostruttiva (da Behn 1934)

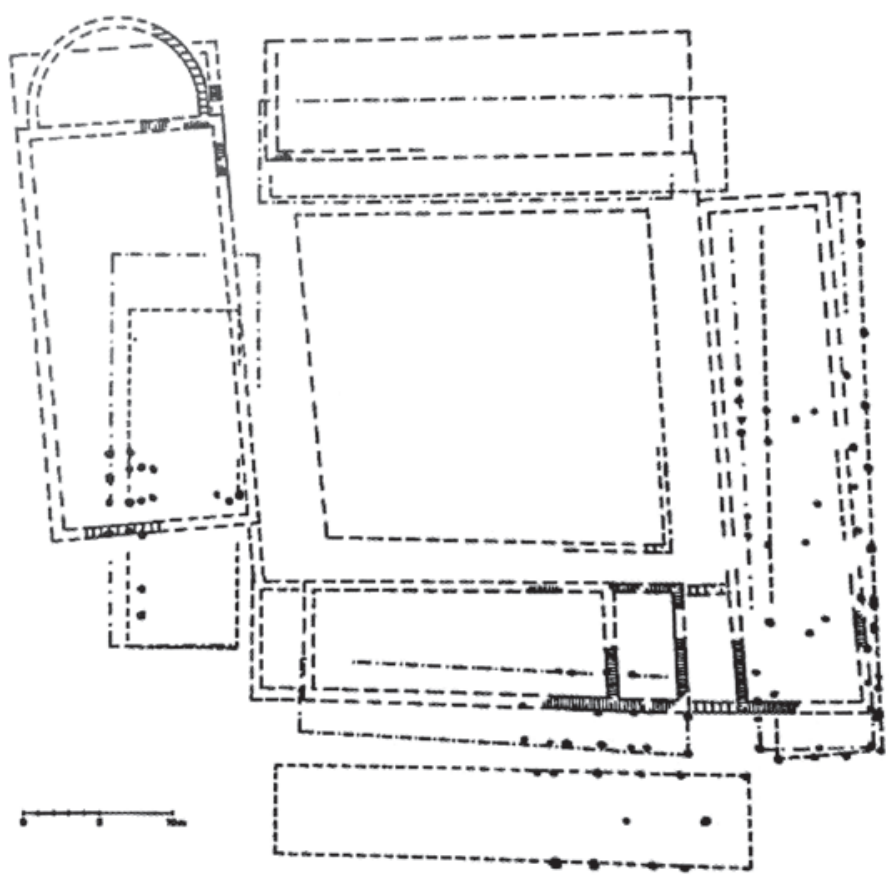

Fig. 14 Herrenchiemsee, planimetria (da Dannheimer 2010)

A primi del IX secolo, l'organismo porticato fu replicato nella ricostruzione di Lorsch su un sito poco distante dall'Altenmüster al tempo dell'abate Richbod (784-804), dove gli scavi hanno riportato alla luce tracce di due lunghi ambienti rettangolari, interpretati come dormitorio (a est) e refettorio (a ovest), quest'ultimo scandito in due navate da un colonnato centrale ${ }^{31}$ (fig. 15). All'incirca nella stessa epoca il dispositivo fu perfezionato anche a Reichenau, sebbene interessò di nuovo solo la Mittelzell ${ }^{22}$ (fig. 12), mentre nelle altre fondazioni dell'isola non ve ne è traccia, a testimonianza dell'assenza di qualsiasi valore prescrittivo in tale schema progettuale.

Del resto anche la posizione laterale del chiostro, a nord o a sud dell'abbaziale, non rappresentava ancora una costante. Nella Inden Kornelimünster anianea (fig. 16) si disponeva di fronte al prospetto dell'abbaziale, citando da vicino gli atri delle basiliche paleocristiane ${ }^{33}$ e, nel caso di Fulda, le ali porticate aggiunte dall'abate Eigel $(817-822)$ alla basilica di Ratgar segnano una curiosa preferenza retrocorale, con un utilizzo per scopi assembleari della galleria tangente l'emiciclo absidale che riecheggia, come già Reichenau, il Piano di San Gallo, ma che questa volta si motiva con la volontà dei monaci di radunarsi per il capitolo presso la tomba del fondatore ${ }^{34}$ (fig. 17).

${ }^{31}$ F. BEHN, op. cit. (n. 24), ma per una sintesi dei risultati degli scavi susseguitisi nel sito fino ad anni recenti si rimanda a B. PINSKER, Kloster Lorsch, Kloster Altenmünster und Kloster Hagen. Eine Forschungsgeschichte, in Kloster Lorsch. Vom Reichskloster Karls des Großen zum Weltkulturerbe der Menschheit. Austellung Museumszentrum Lorsch 28.5.2011 - 29.1.2012, Petersberg, 2011, p. 90-115.

${ }^{32} \mathrm{Nel}$ nuovo assetto la sala riscaldata fu spostata nell'ala ovest dove rimase anche nei secoli a venire, v. A. ZETTLER, Die Konventbauten, op. cit. (n. 23 ), p. 276. 33 L. HUGOT, Kornelimünster. Untersuchung über die baugeschichtliche Entwicklung der ehemaligen Benediktinerklosterkirche, Köln-Graz, 1968 (Beihefte der Bonner Jahrbücher, 26).

${ }^{34}$ P.F. PISTILLI, R. LEGLER, W. JACOBSEN, op. cit. (n. 2), p. 22; W. JACOBSEN, Die Abteikirche in Fulda von Sturmius bis Eigil - kunstpolitische Positionen un deren Veränderungen, in G. Schrimpe (ed.), Kloster Fulda in der Welt der Karolinger und Ottonen, Frankfurt a.M., 1996, p. 105-127. La posizione retrocorale 


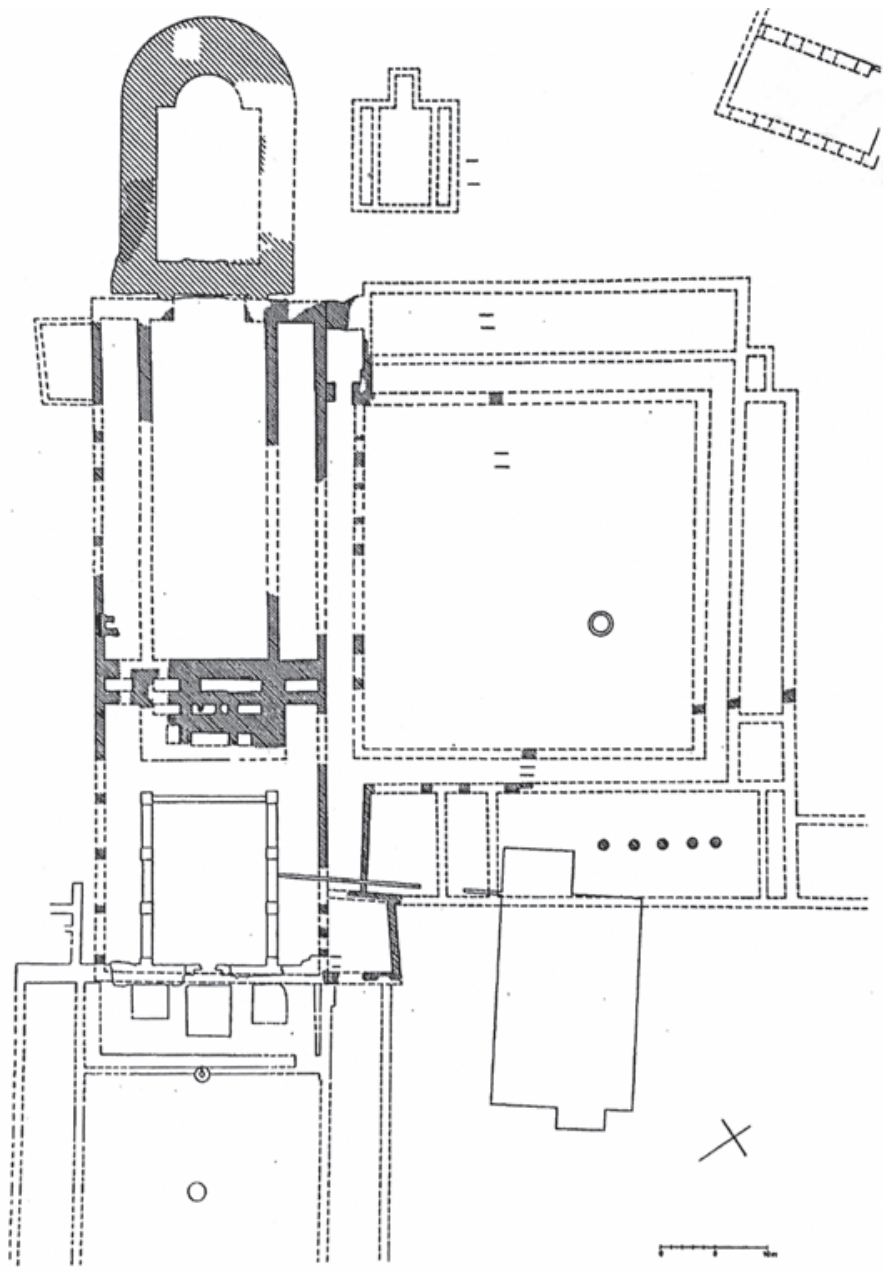

Fig. 15 Lorsch II, planimetria (da Behn 1934)

Ancora diversa, e non meno singolare, la scelta planimetrica operata a Müstair dove il quadrangolo porticato si congiunge alla chiesa con un unico punto di contatto nello spigolo sud-ovest di quest'ultima ${ }^{35}$ (fig. 18). La distribuzione delle strutture residenziali è anomala e la lettura funzionale degli spazi difficoltosa, considerata anche la peculiare destinazione palaziale dell'ala settentrionale e la presenza di complessi percorsi di collegamento con un sistema di corridoi e annessi laterali alla chiesa che costituisce quasi un unicum. Del tutto inusuale è la presunta posizione della sala capitolare vicina all'annesso laterale nord dell'abbaziale e controversa è finanche la collocazione del dormitorio e del refettorio, con plausibilità sovrapposti nel blocco est ${ }^{36}$.

L'adozione diffusa del chiostro nelle abbazie carolingie è confermata anche dalle fonti che, non senza qualche ambiguità, lo attestano in altri contesti oltre a quelli indagati dall'archeologia. Emblematico in questo senso l'esempio di Fontenelle, la cui cronaca rievoca la rifondazione al tempo

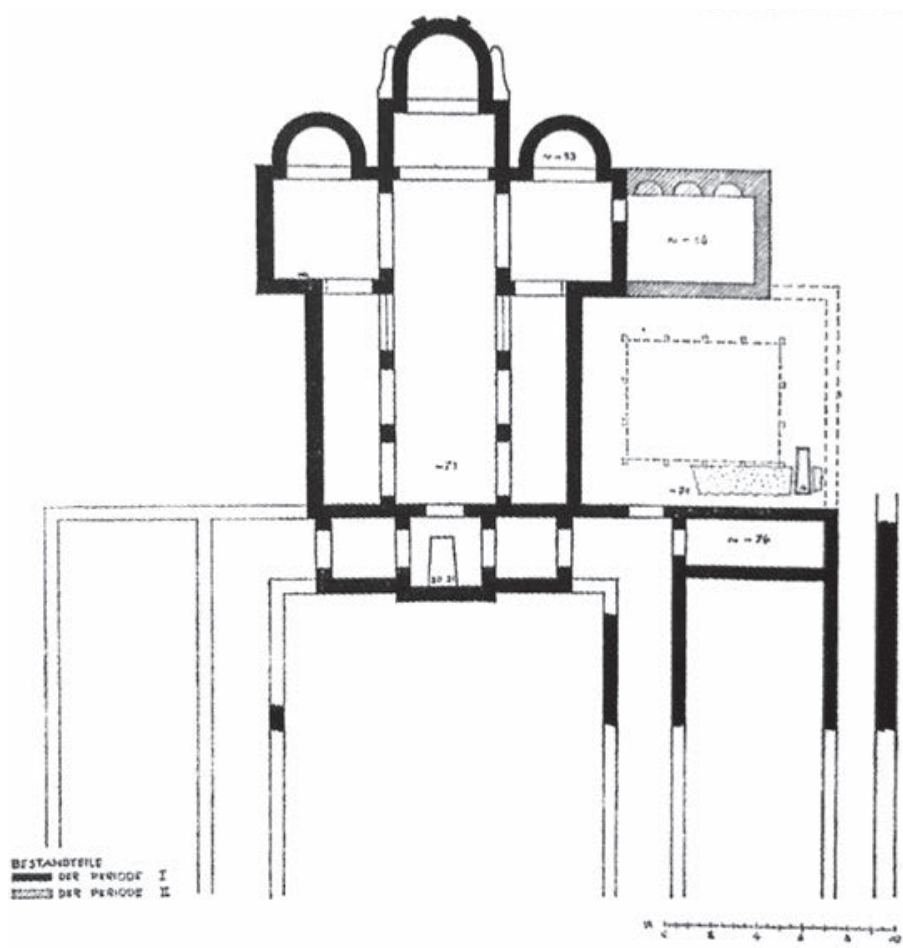

Fig. 16 Inden, planimetria (da Hugot 1965)

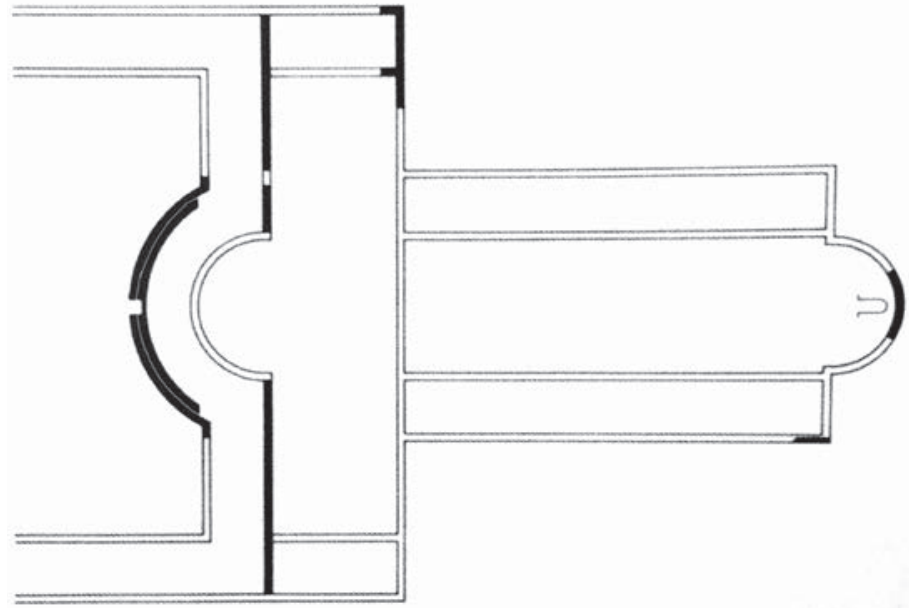

Fig. 17 Fulda, planimetria dell'abbaziale nell'819 (da McClendon 1987)

dell'abate Ansegiso (tra 823 e 834) con una descrizione del complesso di edifici, questa volta a un solo piano, che dovevano serrare ali porticate: il dormitorum fratruum nobilissimum, l'alia domus alloggiante il refettorio e il cellario, una domus maior con camara e caminata e altri ambienti ancora $^{37}$. Discordanti sono le ipotesi ricostruttive avanzate sulla collocazione reciproca delle diverse fabbriche (figg. 1920), ma in ogni caso dal testo si evincono chiaramente sia la

della corte di Fulda trova confronto nella planimetria di Farfa al tempo di Sicardo (830-842), v. C.B. MCCLENDON, The Imperial Abbey of Farfa. Architectural currents of the early Middle Ages, New Haven and London, 1987, p. 66-71.

${ }^{35}$ La collocazione del chiostro ad un angolo della facciata trova rispondenza nel caso di Santa Giulia a Brescia, oscillante dal punto di vista cronologico tra l'età tardolongobarda e, più plausibilmente, quella carolingia, cfr. G.P. BROGIOLO, Gli edifici monastici nelle fasi altomedievali, in R. Stradiotti (ed.), San Salvatore-Santa Giulia a Brescia. Il monastero nella storia, Milano, 2001, p. 64-66; G. ARCHETTI, "Secundum monasticam disciplinam". San Salvatore di Brescia e le trasformazioni istituzionali di un monastero regio, in G. Archetti (ed.), Desiderio. Il progetto politico dell'ultimo re longobardo, Atti del Primo convegno internazionale del Centro studi longobardi (Brescia, 21-24 marzo 2013), 2 voll., Spoleto-Milano 2015 (Centro studi longobardi. Convegni 1, 1.2), I, pp. 631-680; F. STROPPA, La basilica di San Salvatore: i cicli pittorici altomedievali. Per una rilettura del complesso monastico di Santa Giulia di Brescia, Ibidem, II. ${ }^{36}$ Sul problema planimetrico di Müstair si è espresso H.R. SENNHAUSER, Funktionale Bestimmung von Trakten und Räumen der Karolingischen Klosteranlage von Müstair, in H.R. Sennhauser (ed.), op. cit. (n. 9). Per il refettorio di recente si è ipotizzata anche la presenza nell'ala est, v. ID., St. Johann in Müstair als Klosterpfalz, in Pfalz - Kloster - kloster-pfalz, op. cit. (n. 28), p. 3-28.

${ }_{37}$ S. LOEWENFELD (ed.), Gesta abbatum Fontanellensium, Hannoverae, 1886, (Scriptores Rerum Germanicarum, 28), p. 54-56. 


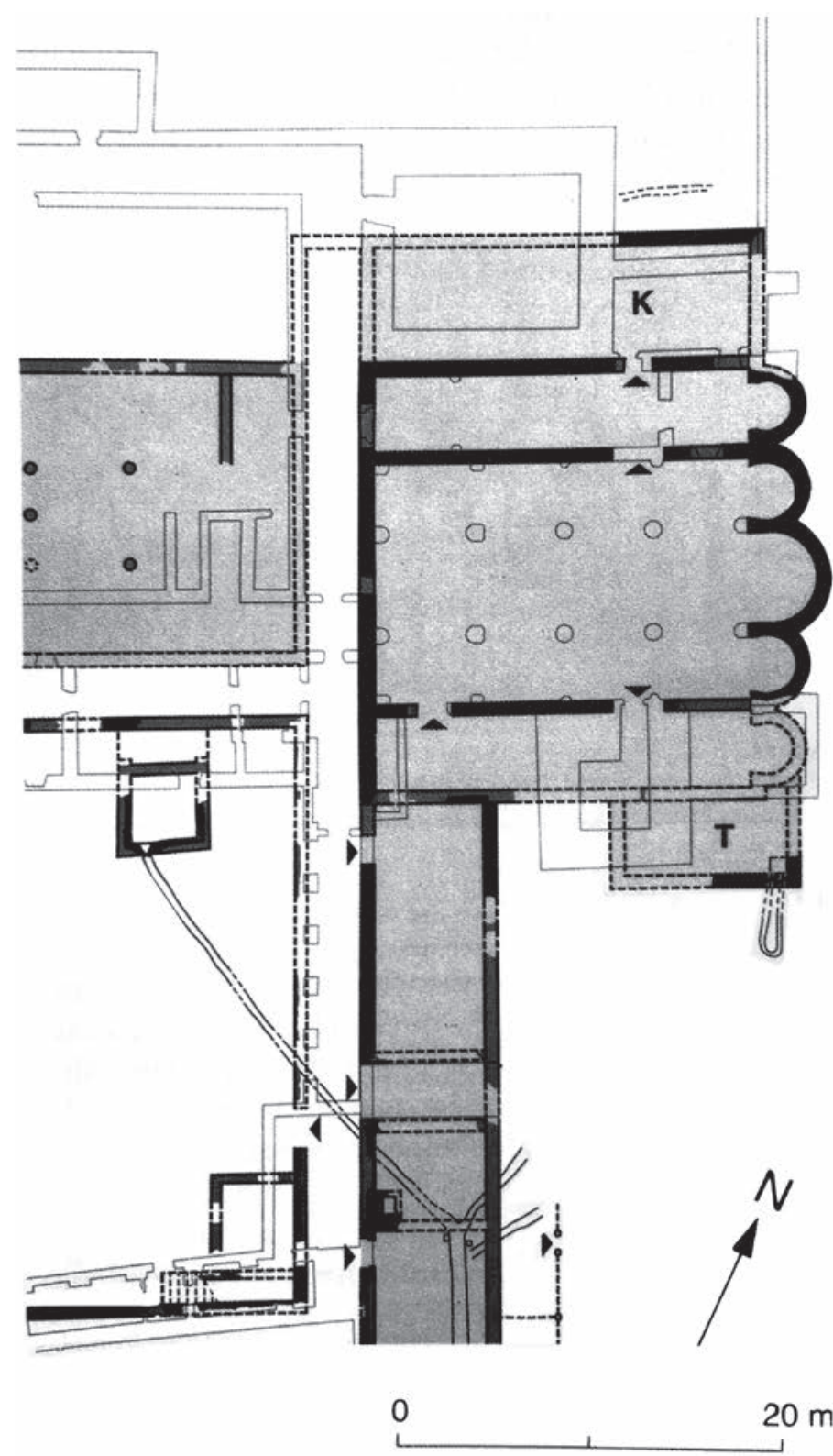

Fig. 18 Müstair (da Sennahauser 1996)

presenza di portici, sia alcune peculiarità nella distribuzione spaziale degli ambienti $3^{8}$ : la scomoda ubicazione del dormitorio lontano dal coro, la coabitazione del refettorio e della dispensa nello stesso blocco, nonché il controverso utilizzo della galleria adiacente la chiesa per l'officio del capitolo o, piuttosto, l'esistenza precoce di una sala capitolare autonoma (bouleuterion) 39 .

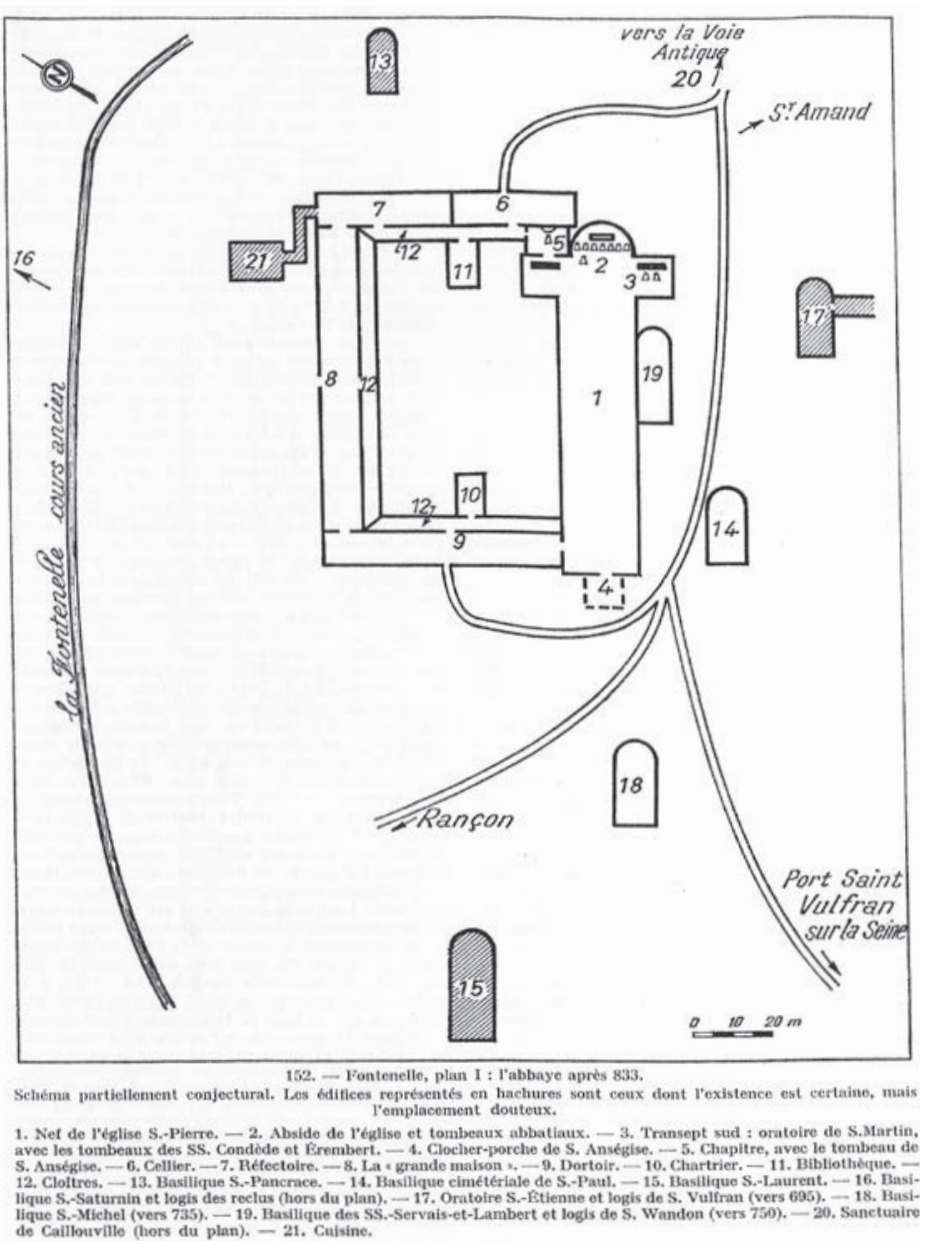

Fig. 19 Fontenelle, ipotesi ricostruttiva (da Laporte 1970)

L'affermazione del dispositivo claustrale non concluse la sperimentazione progettuale in atto nei cantieri monastici, che si protrasse per tutto il X secolo, in particolare per quanto concerne la topografia degli ambienti residenziali, tutt'altro che canonizzata. Una prima normalizzazione in questo senso avvenne intorno al 1000 nell'ambito del cantiere di Cluny (fig. 21) il cui chiostro fu impostato durante il governo di Maiolo (948-991) e poi rifondato da Odilone (994-1048) ${ }^{40}$ e che da un lato, come si vedrà, ripropose scelte planimetriche e progettuali già comparse in età altomedievale, dall'altro, rinnovò il prototipo conferendogli un'eccezionale veste formale di cui oggi percepiamo il riflesso nella sua folta discendenza che si dipanò lungo tutta la stagione romanica (fig. 22). La rinnovata affermazione del modello coincise ancora una volta, e non a caso, con una fase di revisione della vita monastica e, sulla scia della riforma di Cluny, nonché di quella correlata di Hirsau ${ }^{41}$,

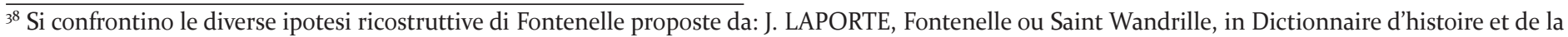
géographie ecclésiastique, XVII, Paris, 1970, coll. 915-953: 941-942; W. HORN, E. BORN, op. cit. (n. 18), II, p. 278. Si segnala anche la recentissima restituzione di F. MARAZZI, Refettori e refezione nei monasteri altomedievali: uno sguardo attraverso l'archeologia e le fonti scritte, in L. ERMINI PANI, op. cit. (n. 4), p. 329-369: 342, fig. 4, dove si ipotizza la disposizione retrocorale dell'abitato monastico che risolverebbe l'incongruente in apparenza lontananza del dormitorio dal presbiterio.

${ }^{39}$ S. LOEWENFELD (ed.), op. cit. (n. 37), p. 6o. Alla fine della vita di Ansegiso l'autore informa che l'abate è stato tumulato: "extra basilicam sancti Petri ad aquilonalem plagam, in porticu, in qua fratres conventum celebrare soliti sunt" e dunque attesta la presenza di portici, ma fornisce un'ulteriore collocazione per la riunione capitolare rispetto a quanto asserito all'inizio, v. R. CERONE, op. cit. (n. 19), p. 53-54.

$4^{\circ}$ K.J. CONANT, Cluny. Les églises et la maison du chef d'ordre, Cambridge (MA)-Mâcon, 1968. Le ipotesi di Conant sono state in parte riviste alla luce degli scavi degli anni Novanta e della campagna 2006-2010, v. A. BAUD, G. ROLLIER, Liturgie et espace monastique à Cluny à la lecture du Liber Tramitis, «Descriptione monasterii» et données archéologiques, in A. Baud (ed.), Espace ecclésial et liturgie au Moyen Âge, Lyon, 2010, (Travaux de la maison de l'Orient e de la Méditérranée, 53), p. 27-42; A. BAUD, CH. SAPIN, Les fouilles de Cluny:état des recherches récentes sur le début du monastère et ses églises, Cluny I et Cluny II, in D. Iogna-Prat, M. Lauwers, F. Mazel, I. Rosé (eds.), Cluny. Les moines et la société au premier âge féodal, Rennes, 2013, p. 497-514, ${ }^{41} \mathrm{~J}$. WOLLASCH, Cluny und Deutschland, in Studien und Mitteilungen zur Geschichte des Benediktinerordens und seiner Zweige, 103, 1992, p. 7-32; M. UNTERMANN, Cluny et l'Empire, in N. Stratford (ed.), Cluny 910-2010. Onze siècles de rayonnement, Paris, 2010, p. 302-311. 


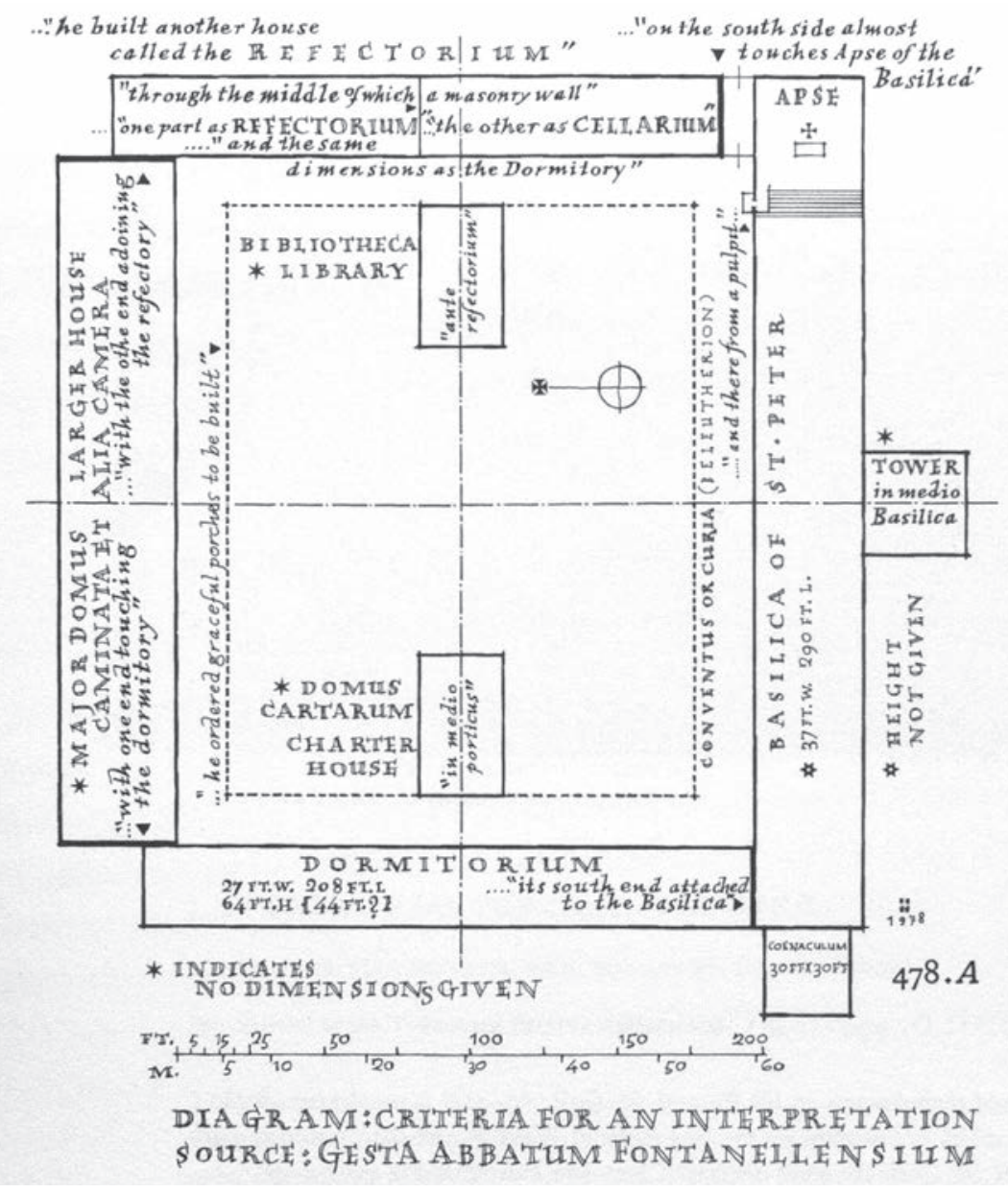

Fig. 20 Fontenelle, ipotesi ricostruttiva (da Horn, Born 1979)

si registrerà un capillare adeguamento all'impianto, con l'eccezione dei centri di minori dimensioni dove si constata un uso del chiostro alquanto morigerato ben oltre l'XI secolo ${ }^{42}$.
La misura del cambiamento emerge attraverso le differenti versioni delle consuetudini cluniacensi che registrano con precisione le modalità di utilizzo delle gallerie ${ }^{43}$. Già nelle Antiquiores, redatte a cavallo dell'anno Mille, ci si dilunga su modi e tempi della lectio, l'attività principale da tenersi lungo gli ambulacri ${ }^{4}$. Ulteriori elementi emergono nel Liber Tramitis (1024-1048) ${ }^{45}$, nell'Ordo cluniacensis di Bernardo di Cluny ${ }^{46}$ e nelle consuetudini di Guglielmo di Hirsau ${ }^{47}$, risalenti al 108o circa, e nella tarda versione delle Floriacenses, oramai pienamente duecentesca ${ }^{48}$. Il chiostro si conferma come il luogo privilegiato per la lectio divina e allo scopo vi è talvolta collocato un armariolum per contenere i libri per la lettura ${ }^{49}$. Dopo l'ora Prima e dopo la refezione, sia a pranzo che a cena, ci si sedeva a leggere: gli infantes leggevano e poi cantavano ad alta voce, i confratelli invece con voce sommessa ${ }^{50}$.

Nelle gallerie era consentito anche il colloquio, purché di argomenti virtuosi e in momenti precisi, come dopo l'officio capitolare ${ }^{51}$, ma era possibile conversare solo nei giorni stabiliti, altrimenti occorreva rispettare un rigoroso silenzio, in particolare durante la settimana santa. Fa eccezione il venerdì di Parasceve, nel momento subito dopo Prima, quando la comunità si raduna nel chiostro per cantare i salmi ${ }^{2}$.

Emerge poi che il chiostro, per la presenza del lavatorium e dei manutergia ${ }^{53}$, è il luogo deputato all'igiene, personale o degli oggetti privati, ma alle abluzioni e ai lavaggi ci si può dedicare solo nelle ore in cui è ammesso il colloquio. Nel chiostro ogni monaco deterge la iustitiam o il proprio scyphum ${ }^{54}$, nonché le calzature e gli abiti che possono poi essere stesi ad asciugare sullo scamnum vicino il cellarium 55 . Qui il cuoco lava i

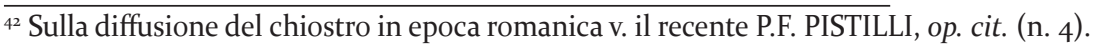

${ }_{43}$ Una prima disamina delle fonti monastiche per la definizione degli utilizzi del chiostro dopo il 10oo è in P. MEYVAERT, The medieval monastic claustrum, in Gesta, 12, 1973, p. 53-59; A. DAVRIL, Fonctions des cloîtres dans les monastères du Moyen Age, in P.K. KLEIN (ed.), op. cit. (n. 3), p. $22-26$.

${ }^{44}$ K. HALLIGER (ed.), Consuetudines cluniacensium antiquiores cum redactionibus derivatis, in K. Halliger (ed.), Corpus consuetudinum monasticarum, $\mathrm{VII} / 2$, Siegburg, 1983.

${ }^{45}$ P. DINTER (ed.), Liber tramitis aevi Odilonis abbatis, in K. Halliger (ed.), Corpus consuetudinum monasticarum, X, Siegburg, 1980.

${ }^{46}$ BERNARDUS CLUNIACENSIS, Ordo cluniacensis, in M. Herrgott (ed.), Vetus disciplina monastica, Parisiis, Typis Carolis Osmont, 1726, p. 134-364.

${ }^{47}$ WILHELMUS HIRSAUGIENSIS, Constitutiones hirsaugienses seu gengebacenses, Ibidem, p. 375-570.

${ }^{48}$ A. DAVRIL (ed.), Consuetudines floriacenses saeculi tertii decimi, in K. Halliger (ed.), Corpus consuetudinum monasticarum, IX, Siegburg, 1976.

${ }_{49}$ BERNARDUS CLUNIACENSIS, op. cit. (n. 46), p. 212. L'armariolum, consistente in una piccola nicchia ricavata nella parete claustrale, è ancora esistente a Fleury, v. A. DAVRIL, op. cit. (n. 43), p. 23.

${ }^{50}$ K. HALLIGER (ed.), op. cit. (n. 44), p. 10: "infantes vero debent aut legere aut cantare excelsa voce. Primitus enim debent legere infantes et postea canant. Fratres autem sub silentio legant vel cantent”. Sulla presenza degli oblati, G. ARCHETTI, «Sub virga magistri». Custodia e disciplina nell'educazione carolingia dei pueri oblati, in Studi medievali, terza serie, LVII, 2, 2016, p. 527-582.

${ }^{51}$ Ibidem, p. 54: "Post capitulum vero non debent loqui in claustra fratres, id est, feria secunda et feria sexta et feria septima. Aliis autem tribus diebus loquantur in claustra". I giorni del silenzio dopo il capitolo variano nelle differenti versioni; secondo il Liber tramitis, ad esempio, "post capitulum non loquantur in claustro secunda, quarta et sexta feria", v. P. Dinter (ed.), op. cit. (n. 45), p. 57.

${ }^{52}$ K. HALLiGER (ed.), op. cit. (n. 44), p. 88, 91, 95. Nell'ambito dei rituali di Parasceve dopo l'ora Nona e in particolare dopo l'Adorazione della Croce nel coro: "in hunum in claustra simul cantent psalterium totum usque in fine". Anche secondo quanto tramanda il Liber tramitis, c'è il medesimo rituale ma da svolgersi dopo l'ora Prima: "in Parasceve post Primam exeuntes communiter fratres dicant in claustrum psalterium ex integro". Guglielmo di Hirsau dedica un intero capitolo alle raccomandazioni sulle attività da compiere in tempore loquendi e in particolare si raccomanda: "Quod loquitur, qui loquitur, submissa voce. Loquela sua et audentia non sit, nisi de spiritualibus vel de his, quibus haec vita temporalis carere non potest, Cavet etiam loqui ad alium fratrem in opposito, vel remotiori loco sedentem aut stantem”, v. WILHELMUS HIRSAUGIENSIS, op. cit. (n. 47), p. 430.

53 BERNARDUS CLUNIACENSIS, op. cit. (n. 46), p. 175.

${ }^{54}$ Ibidem, p. 215 ss. C'è un intero capitolo dedicato all'igiene nel chiostro: De opere manu que fit in claustro. Anche Guglielmo di Hirsau si occupa della questione e precisa: "Si opus habet iustitiam suam et pateram lavare, tunc lavat observato ibi silentio", v. WILHELMUS HIRSAUGIENSIS, op. cit. (n. 47), p. 430. 55 Ibidem, p. 530: "omni sabbato post Nonam et in Quadragesima post coenam, panni fratrum noviter abluti ponuntur in claustro super scamnum, quod est prope cellarium".
} 


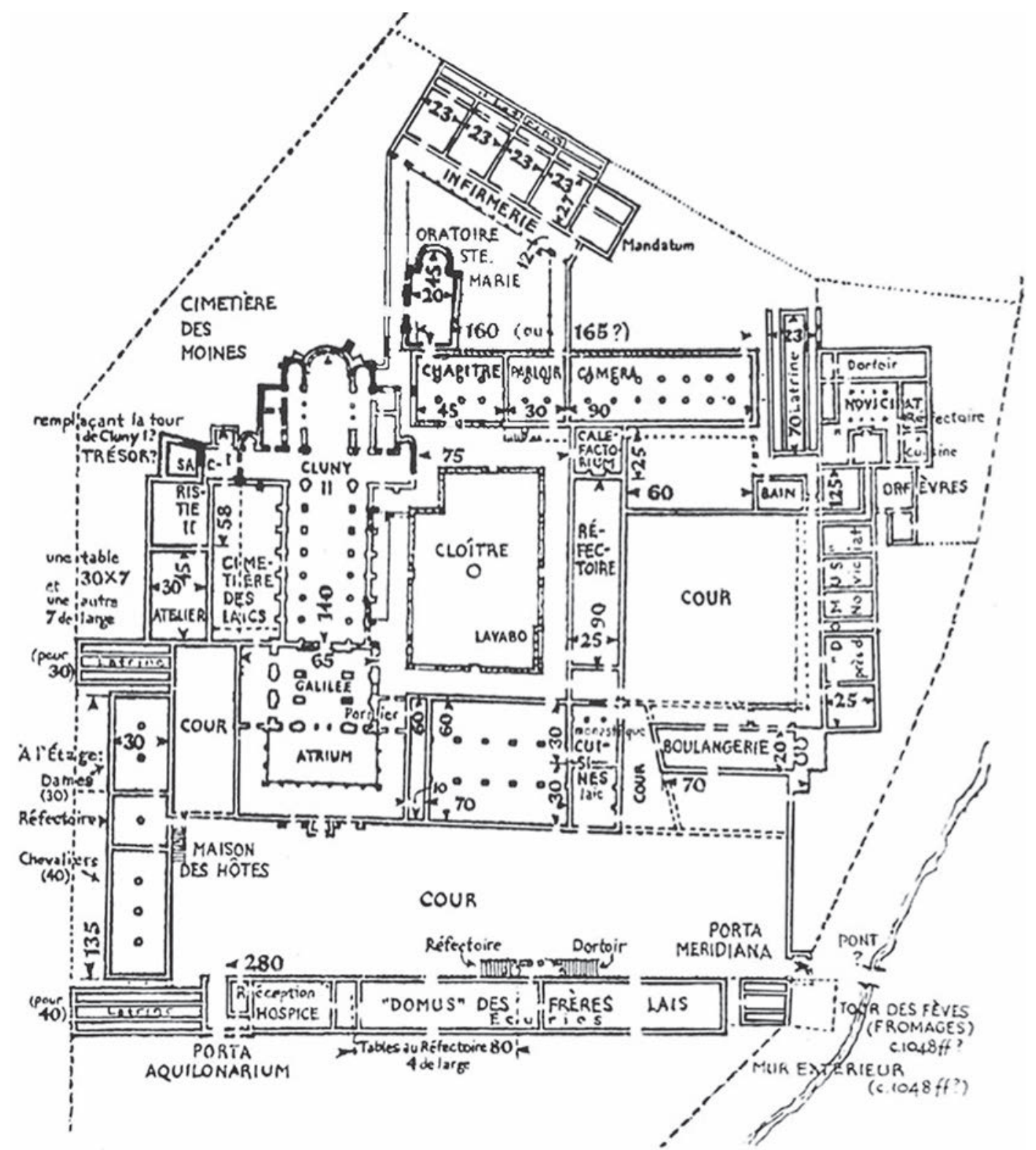

Fig. 21 Cluny, planimetria del monastero al tempo di Odilone (da Conant 1968)

panni che si usano in cucina, ogni monaco affila il proprio coltello e provvede alla rasatura periodica di barba e capelli e al taglio delle unghie ${ }^{56}$. Il chiostro stesso deve mantenersi ordinato e pulito ${ }^{57}$ e a questo scopo il circator esegue le ronde dopo completorio per verificare che tutto sia in ordine, mentre al monaco elemosiniere spetta il recupero degli oggetti perduti e la pulizia delle gallerie ${ }^{58}$.

Compaiono quindi indicazioni sulla differenziazione funzionale di alcune sezioni degli ambulacri, finalizzata a non creare confusione nei momenti di passaggio tra un'attività e l'altra e a evitare sovrapposizioni tra gruppi di monaci e di novizi. In alcuni casi, infatti, a questi ultimi è riservato un settore diverso dai confratelli, dotato di sgabelli (truncos) di legno opportunamente distanziati tra loro59, mentre lo spazio tra il capitolo e il refettorio è denominata trisantia ed è un luogo d'attesa tra un momento liturgico e l'altro: qui i ragazzi con il maestro attendono la fine del capitolo e i fratres si radunano alla fine dei lavori manuali in attesa del priore ${ }^{60}$.

In occasioni particolari le gallerie sono utilizzate anchea scopi liturgici, come in coena Domini quando vi si effettuava il mandatum pauperum, il lavaggio rituale dei piedi dei poveri: i monaci cantavano il salmo 50 e arrivati al Gloria patri si ponevano davanti ai tre indigenti scelti per il rito che erano già stati preparati dai famuli con i piedi nudi e prelavati $^{61}$. Nell'ambulacro adiacente la chiesa si svolgevano le processioni che andavano dalla chiesa all'oratorio di Santa Maria posto a nord del capitolo e quindi di nuovo nel

${ }^{56}$ BERNARDUS CLUNIACENSIS, op. cit. (n. 46), p. 215-216.

${ }^{57}$ Ibidem, p. 239. In questo caso è il cuoco che deve provvedere alla pulizia del chiostro.

${ }^{8}$ P. DINTER (ed.), op. cit. (n. 45), p. 215; BERNARDUS CLUNIACENSIS, op. cit. (n. 46), p. 157-16o.

59 Ibidem, p. 165.

${ }^{60}$ Ibidem, p. 280. Secondo Herrgott la trisantia è la "pars seu ala claustri integra capitulo Ecclesiam refectorio proxima; vocis origo vel a tribus tantum quibus constabat claustrum porticibus".

${ }^{61}$ P. DINTER (ed.), op. cit. (n. 45), p. 75: "Decanus autem claustrensis et decanus forensis cum camerario et etiam de aliis fratribus quibus velint assumere interim, dum in refectorio sunt fratres, in locum quo constitutum est, videlicet in claustro iuxta ecclesia, deducant pauperes ad sedendum”. 
coro $^{62}$. Queste si svolgevano in modalità differenti a seconda delle festività che però mutano nelle diverse fonti: il Liber Tramitis ci informa che si tenevano a Natale, a Pasqua e nel giorno dell'Assunzione; per Bernardo di Cluny invece si facevano il giorno dell'Ascensione, della Purificatio, la domenica delle Palme, la prima domenica d'Avvento e poi tutte le domeniche dalla Septuagesima fino al tempo di Quaresima $^{63}$.

Alla rilevanza funzionale del chiostro che le consuetudini mostrano come tutt'altro che un semplice luogo di passaggio, ma come uno spazio attorno cui ruotava la vita quotidiana della comunità, corrispondevano nel caso di Cluny dimensioni monumentali ${ }^{64}$. Il chiostro dell'abbazia borgognona fu perfezionato su committenza dell'abate Odilone all'inizio dell'XI secolo, in affiancamento all'abbaziale del predecessore Maiolo, la cosiddetta Cluny II, e previde il graduale riassetto dei corpi residenziali che trovarono finalmente una prima canonizzazione. La frammentazione del piano terra del settore est, fino ad allora spesso occupato per intero dal calefactorium, permetteva l'inclusione della sala capitolare in una posizione oramai codificata, mentre limitata alle fondazioni di stretta osservanza cluniacense rimarrà l'innesto sul capitolo della cappella dedicata alla Madonna, destinata ai malati ${ }^{65}$. Il dormitorio vede confermare la sua ubicazione al primo livello del blocco tangente il presbiterio, così come il refettorio nell'ala opposta all'abbaziale, in una formula già prevista nel Piano di San Gallo. Ancora incerti nel piano cluniacense il nodo disarticolato tra parlatorio e calefattorio e la fattura irregolare degli ambulacri che, certo anche a causa della loro sovrapposizione alla precedente fabbrica, mostrano notevoli differenze dimensionali.

Il chiostro stesso aveva un impianto quadrangolare allungato verso ovest con un anomalo gradino nell'angolo nord-est che permetteva l'incastro delle gallerie con il transetto. Della conformazione dei porticati non si conoscono particolari, se non che era scandito da colonnine marmoree, né sopravvivono elementi del corredo plastico, ma il monaco Iotsualdus, biografo di Odilone, ricorda l'ammirazione che il manufatto riscosse presso i contemporanei per la ricchezza dell'apparato scultoreo e l'eleganza dei marmi provenienti dal sud della Francia ${ }^{66}$.

$\mathrm{Fu}$ forse proprio la visione del chiostro cluniacense a scuotere tanto l'animo di Bernardo di Clairvaux, quando nel 1124 scrisse sdegnato la I Epistola al cugino Roberto

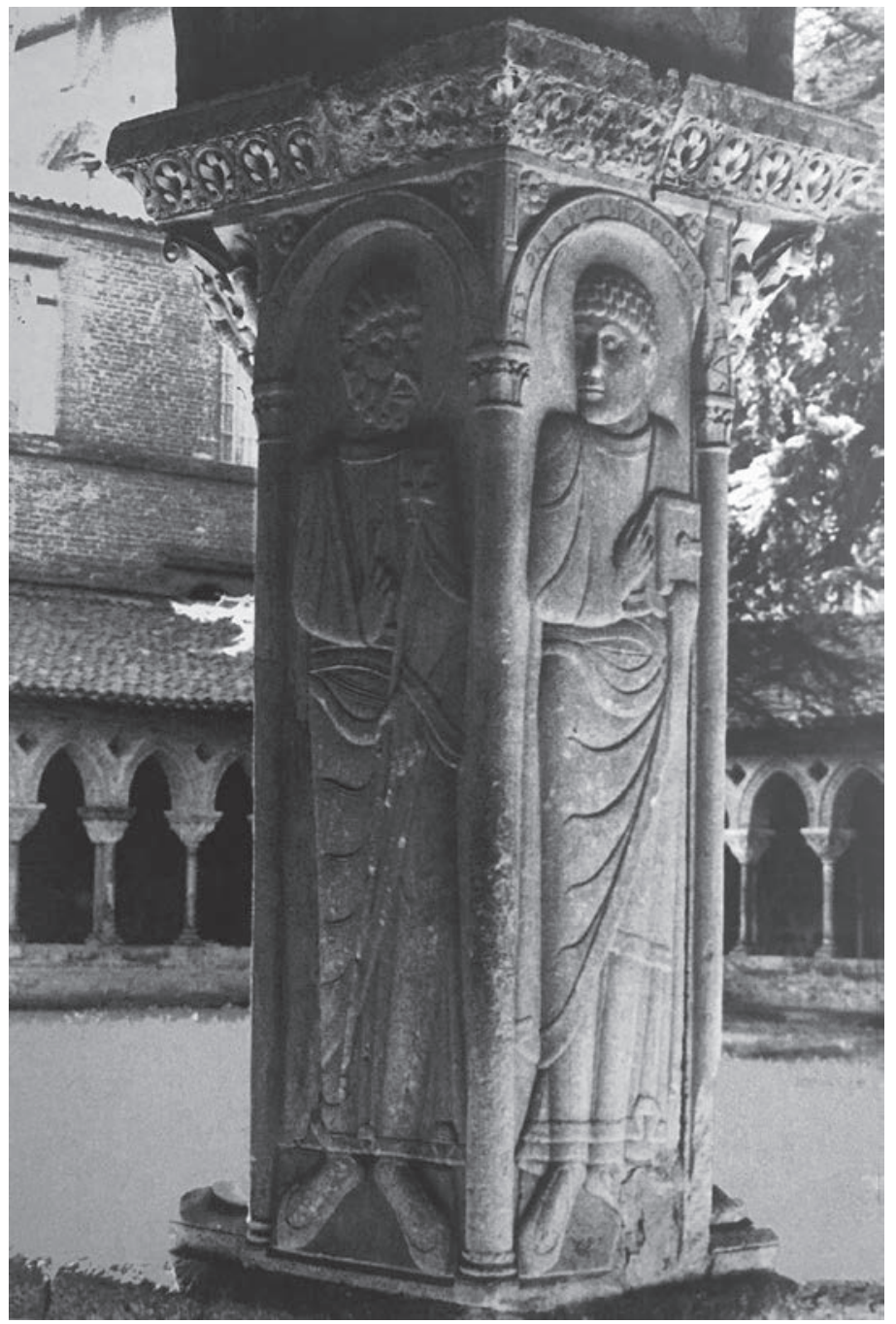

Fig. 22 Moissac, chiostro, part. di un pilastro

criticando le consuetudini dei benedettini di Cluny? È solo una suggestione, per quanto suadente, proposta da Kenneth John Conant ${ }^{67}$, certo è che proprio ai Cistercensi spetterà raccogliere l'eredità di Cluny nella storia dell'architettura monastica segnando la definitiva evoluzione dell'impianto benedettino e la canonizzazione planimetrica del monastero, in un legame tra architettura e norma di vita che nel chiostro trova perfetto compimento.

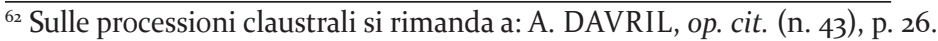

${ }_{63}$ P. DINTER (ed.), op. cit. (n. 45), p. 108, 150; BERNARDUS CLUNIACENSIS, op. cit. (n. 46), p. 300-301, 330.

${ }^{64}$ K.J. CONANT, op. cit. (n. 40), p. 6o-66.

${ }_{5}^{65}$ R. CERONE, op. cit. (n. 19), p. 76. Sull'evoluzione dell'ala dei monaci v. M. UNTERMANN, Das "Mönchshaus in der frü- und hochmittelalterlichen Klosteranlage. Beobachtungen zu Lage und Raumaufteilung des Klausur-Ostflügels, in H.R. Sennhauser (ed.), op. cit. (n. 9), p. 233-257.

${ }^{66}$ K.J. CONANT, op. cit. (n. 40), p. 65: "claustrum costruxit columnis marmoreis per ultimis partibus illius provinciae, ac per rapidissimos Durentiae Rhodanique cursus non sine magno labore advectis, mirabiliter decoratum”.

${ }^{67}$ Ibidem.
} 